\title{
WestVirginiaUniversity
}

THE RESEARCH REPOSITORY @ WVU

Graduate Theses, Dissertations, and Problem Reports

2007

\section{Integer flow and Petersen minor}

\author{
Taoye Zhang \\ West Virginia University
}

Follow this and additional works at: https://researchrepository.wvu.edu/etd

\section{Recommended Citation}

Zhang, Taoye, "Integer flow and Petersen minor" (2007). Graduate Theses, Dissertations, and Problem Reports. 2585.

https://researchrepository.wvu.edu/etd/2585

This Dissertation is protected by copyright and/or related rights. It has been brought to you by the The Research Repository @ WVU with permission from the rights-holder(s). You are free to use this Dissertation in any way that is permitted by the copyright and related rights legislation that applies to your use. For other uses you must obtain permission from the rights-holder(s) directly, unless additional rights are indicated by a Creative Commons license in the record and/ or on the work itself. This Dissertation has been accepted for inclusion in WVU Graduate Theses, Dissertations, and Problem Reports collection by an authorized administrator of The Research Repository @ WVU.

For more information, please contact researchrepository@mail.wvu.edu. 


\title{
Integer Flow and Petersen Minor
}

\author{
Taoye Zhang \\ Dissertation submitted to the \\ Eberly College of Arts and Sciences \\ at West Virginia University \\ in partial fulfillment of the requirements \\ for the degree of \\ Doctor of Philosophy \\ in \\ Mathematics \\ Cun-Quan Zhang, Ph.D., Chair \\ Elaine M Eschen, Ph.D. \\ John Goldwasser, Ph.D. \\ Hong-Jian Lai, Ph.D. \\ Jerzy Wojciechowski, Ph.D. \\ Department of Mathematics \\ Morgantown, West Virginia \\ 2007
}

Keywords: flow, matroid, odd edge connectivity, minor

Copyright 2007 Taoye Zhang 


\section{ABSTRACT \\ Integer Flow and Petersen Minor}

\section{Taoye Zhang}

Tutte [45] conjectured that every bridgeless Petersen-minor free graph admits a nowherezero 4 -flow. Let $\left(P_{10}\right)_{\bar{\mu}}$ be the graph obtained from the Petersen graph by contracting $\mu$ edges from a perfect matching. In chapter 1 we prove that every bridgeless $\left(P_{10}\right)_{\overline{3}}$-minor free graph admits a nowhere-zero 4-flow.

Walton and Welsh [48] proved that if a coloopless regular matroid $M$ does not have a minor in $\left\{M\left(K_{3,3}\right), M^{*}\left(K_{5}\right)\right\}$, then $M$ admits a nowhere zero 4-flow. Lai et al [27] proved that if $M$ does not have a minor in $\left\{M\left(K_{5}\right), M^{*}\left(K_{5}\right)\right\}$, then $M$ admits a nowhere zero 4-flow. We prove in chapter 2 that if a coloopless regular matroid $M$ does not have a minor in $\left\{M\left(\left(P_{10}\right) \overline{3}\right), M^{*}\left(K_{5}\right)\right\}$, then $M$ admits a nowhere zero 4-flow. This result implies Walton and Welsh [48] and Lai et al [27].

The odd-edge-connectivity of a graph $G$, denoted by $\lambda_{o}(G)$, is the size of the smallest odd edge-cut of $G$. In chapter 3, some methods are developed to deal with small even edge-cuts and therefore, extending some earlier results from edge-connectivity to oddedge-connectivity. One of the main results in chapter 3 solves an open problem that every odd- $(2 k+1)$-edge-connected graph has $k$ edge-disjoint parity subgraphs. Another main theorem in the chapter generalizes an earlier result by Galluccio and Goddyn (Combinatorica 2002) that the flow index of every odd-7-edge-connected graph is strictly less than 4. It is also proved in this paper if $\lambda_{o}(G) \geq 4\left\lceil\log _{2}|V(G)|\right\rceil$, then $G$ admits a nowhere-zero 3 -flow which is a partial result to the weak 3-flow conjecture by Jaeger and improves an earlier result by Lai and Zhang[24]. 


\section{Acknowledgements}

I would like to thank my supervisor, Dr. Cun-Quan Zhang, for his guidance, advice, and continual encouragement. It is a pleasure to work under his supervision. Without him, this paper could not have come out.

I would also like to thank my other committee members: Dr. Elaine Eschen, Dr. John Goldwasser, Dr. Hong-Jian Lai and Dr. Jerzy Wojciechowski, for their help during my studies.

And finally, I would like to thank the Department of Mathematics and Eberly College of Arts and Sciences at West Virginia University for providing me with an excellent study environment and support during my study as a graduate student. 


\section{DEDICATION}

To

my father Zuyou, my mother Yonglian

and

my wife $\underline{\mathrm{Ju}}$ 


\section{Contents}

1 Introduction $\quad 1$

1.1 Nowhere-zero 4-flows of graphs . . . . . . . . . . . . . . . 1

1.2 Nowhere-zero 4-flows of matroids . . . . . . . . . . . . . . . . . 3

1.3 Odd-edge-connectivity of graphs . . . . . . . . . . . . . 5

1.3.1 Parity Subgraphs . . . . . . . . . . . . . . 5

1.3.2 Flow Index . . . . . . . . . . . . . . . . . . 6

1.3.3 Nowhere-zero 3-flows . . . . . . . . . . . . . . . . . . . . . . 7

$2 \quad 4-\mathrm{NZF}$ in almost $P_{10}$ minor free graphs $\quad 8$

2.1 Introduction . . . . . . . . . . . . . . . . . 8

2.2 Notations and terminologies . . . . . . . . . . . . . . . . 10

2.3 Lemmas . . . . . . . . . . . . . . . . . . . . . . . . 11

2.4 Proof of the main theorem . . . . . . . . . . . . . . . 13

$3 \quad 4-\mathrm{NZF}$ in regular matroids $\quad 19$ 
3.1 Introduction . . . . . . . . . . . . . . . . . . . . 19

3.2 Decomposition of Regular Matroids in $\operatorname{EX}\left(M\left(K_{5}\right), M^{*}\left(K_{5}\right)\right) \ldots \ldots$

3.3 The Proofs of Theorem 3.1.8 and Corollary 3.1.9 . . . . . . . . . . . 25

4 Odd edge connectivity $\quad 30$

4.1 Introduction . . . . . . . . . . . . . . . . 30

4.1 .1 Parity Subgraphs . . . . . . . . . . . . . . . . 31

4.1 .2 Flow Index . . . . . . . . . . . . . . . . . 32

4.1.3 Nowhere-zero 3-flows . . . . . . . . . . . . . . . . 33

4.2 Notations and Lemmas . . . . . . . . . . . . . . . . . . . . . 33

4.3 Proof of Theorem $4.1 .3 \ldots \ldots \ldots \ldots$. . . . . . . . . . . . . . . . . . .

4.4 Proof of Theorem $4.1 .7 \ldots \ldots \ldots \ldots \ldots$

4.5 Proof of Theorem $4.1 .9 \ldots \ldots \ldots \ldots$. . . . . . . . . . . . . . 42

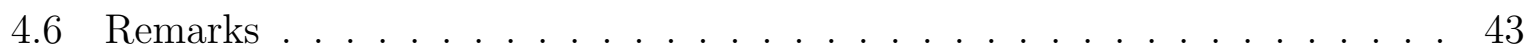




\section{Chapter 1}

\section{Introduction}

\subsection{Nowhere-zero 4-flows of graphs}

The concept of integer flow was introduced by Tutte as a generalization of map coloring problem. For terms that are not defined here, readers can refer to textbooks [5] or [?] for graphs, [54] for flows, and [30] or [50] for matroids.

A nontrivial 2-regular connected graph will be called a circuit, and a disjoint union of circuits a cycle. Thus the empty set $\emptyset$ is the only independent cycle.

Let $G=(V, E)$ be a graph with vertex set $V$ and edge set $E$ and let $D$ be an orientation of $G$. For a vertex $v \in V(G)$, let $E^{+}(v)$ (or $E^{-}(v)$ ) be the set of all arcs of $D(G)$ with their tails (or heads, respectively) at the vertex $v$. $G$ is said to admit a nowhere-zero $k$-flow if there exists an ordered pair $(D, f)$, where $f: E(G) \rightarrow\{ \pm 1, \pm 2, \cdots \pm(k-1)\}$ such that

$$
\sum_{e \in E^{+}(v)} f(e)=\sum_{e \in E^{-}(v)} f(e)
$$

for every vertex $v \in V(G)$.

Let $G$ and $H$ be two graphs. If $G$ contains a subgraph which is contractible to $H$, then $H$ is a minor of $G$ and we say $G$ contains an $H$-mimor. 
The following conjecture is one of the major open problems in graph theory.

Conjecture 1.1.1 (Tutte [45]) Every bridgeless graph without a Petersen minor admits a nowhere-zero 4-flow.

For planar graphs, admitting a nowhere-zero 4-flow is equivalent to having a face 4coloring. Hence, by the 4-Color Theorem [1, 2, 3, 31], Conjecture 2.1.1 has been verified for all planar graphs. Furthermore, it was also announced that Conjecture 2.1.1 was verified for all cubic graphs [32, 33]. By Kuratowski Theorem, a graph is planar if and only if it contains neither $K_{5}$-minor nor $K_{3,3}$-minor. By applying the 4-Color Theorem, Conjecture 2.1.1 was further verified for $K_{3,3}$-minor free graphs [48], $K_{5}$-minor free graphs [27], and $P_{10}^{-}$-minor free graphs [39]. Each of these families contains the family of all planar graphs and may not be necessarily cubic. Graphs $K_{5}, K_{3,3}, P_{10}$ and $P_{10}^{-}$are illustrated in the following figure.

Figure 1:

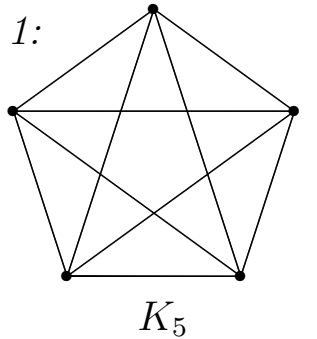

Figure 2:

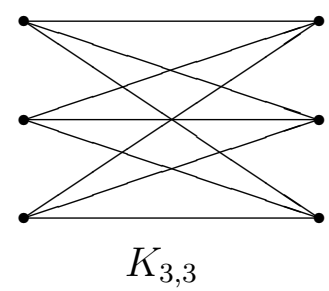

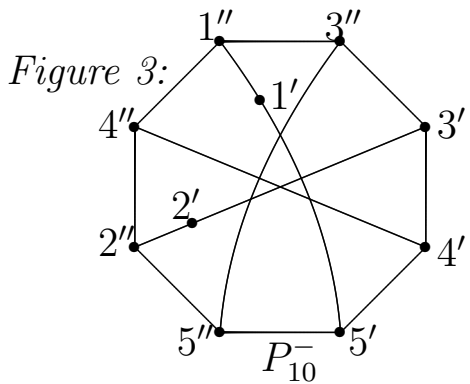

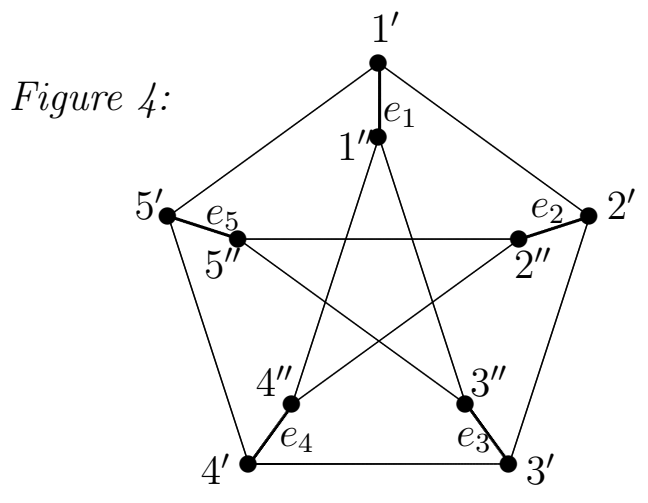

Petersen graph $P_{10}$

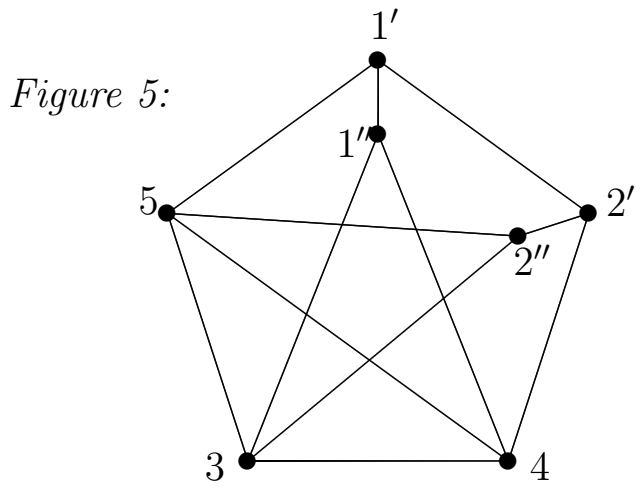

$\left(P_{10}\right)_{\overline{3}}=P_{10} /\left\{e_{3}, e_{4}, e_{5}\right\}$

Let $P_{10}$ be the Petersen graph with the exterior pentagon $1^{\prime} 2^{\prime} 3^{\prime} 4^{\prime} 5^{\prime} 1^{\prime}$, interior pentagon $1^{\prime \prime} 3^{\prime \prime} 5^{\prime \prime} 2^{\prime \prime} 4^{\prime \prime} 1^{\prime \prime}$ and a perfect matching $M=\left\{e_{i}=i^{\prime} i^{\prime \prime}: i=1,2,3,4,5\right\}$. Let $\left(P_{10}\right)_{\bar{\mu}}$ be the 
graph obtained from $P_{10}$ by contracting $F$, where $F \subseteq M$ and $|F|=\mu$.

Remark. It is not hard to see that if $M$ and $M^{\prime}$ are two perfect matchings of $P_{10}$, $F \subseteq M, F^{\prime} \subseteq M^{\prime}$ and $|F|=\left|F^{\prime}\right|$, then $P_{10} / F \cong P_{10} / F^{\prime}$. Hence $\left(P_{10}\right)_{\bar{\mu}}$ is well defined.

The following is the main theorem of this section and will be proved in chapter 2 .

Theorem 1.1.2 Let $G$ be a bridgeless graph. If $G$ does not have a $\left(P_{10}\right) \overline{3}$-minor, then $G$ admits a nowhere-zero 4-flow.

\subsection{Nowhere-zero 4-flows of matroids}

We shall assume familiarity with graph theory and matroid theory. For terms that are not defined in this note, see Bondy and Murty [5] for graphs, and Oxley [30] or Welsh [50] for matroids.

In this article, $\mathbf{Z}, \mathbf{Z}^{+}$and $\mathbf{Z}_{n}$ denote the additive group of the integers, the set of all positive integers, and the cyclic group of order $n$, respectively, and $\mathcal{R}$ denotes the family of all regular matroids. As in [30], the set of all circuits of a matroid $M$ is denoted by $\mathcal{C}(M)$. We further denote the set of all cycles of a matroid $M$ by $\mathcal{C}_{0}(M)$. Note that as we allow empty unions, the empty set is also a cycle (in both graphs and matroids). For matroids $N_{1}, N_{2}, \cdots, N_{k}$, let $E X\left(N_{1}, N_{2}, \cdots, N_{k}\right)$ denote the collection of matroids such that a matroid $M \in E X\left(N_{1}, N_{2}, \cdots, N_{k}\right)$ if and only if $M$ does not have a minor isomorphic to any one in $\left\{N_{1}, N_{2}, \cdots, N_{k}\right\}$. The Fano matroid $F_{7}$ is the vector matroid over $\mathrm{GF}(2)$ of the following matrix $A$ :

$$
A=\left[\begin{array}{lllllll}
1 & 0 & 0 & 1 & 1 & 0 & 1 \\
0 & 1 & 0 & 1 & 0 & 1 & 1 \\
0 & 0 & 1 & 0 & 1 & 1 & 1
\end{array}\right]
$$

The definition of flow has a natural extension to regular matroids. Let $M$ be a regular matroid and $D_{M}$ be its incidence matrix of circuits against elements. An orientation 
$\left(w\left(D_{M}\right), w\left(D_{M^{*}}\right)\right)$ is an assignment of,+- signs to the "1" entries of $D_{M}$ and $D_{M^{*}}$, respectively, so that the resulting matrices $w\left(D_{M}\right)$ and $w\left(D_{M^{*}}\right)$ satisfy

$$
w\left(D_{M}\right) w\left(D_{M^{*}}\right)^{T}=0
$$

Let $A$ be an abelian group. For an element $a \in A$, and for integers $+1,-1,0$, we adopt the convention to write $(+1) \cdot a=a,(-1) \cdot a=-a$ and $0 \cdot a=0$. Let $F^{*}(M, A)=$ $\{f: E(M) \mapsto A \backslash\{0\}\}$ denote the set of all functions from $E(M)$ into $A \backslash\{0\}$. A map $f \in F^{*}(M, A)$ can be viewed as an $|E(M)|$-dimensional column vector. For a regular matroid $M$ with an orientation $\left(w\left(D_{M}\right), w\left(D_{M^{*}}\right)\right)$, a map $f \in F^{*}(M, A)$ satisfying

$$
w\left(D_{M^{*}}\right) \cdot f=0
$$

is a nowhere zero $A$-flow ( $A$-NZF for short) of $M$. When $A=\mathbf{Z}$, a Z-NZF $f$ of $M$ is called a nowhere zero $k$-flow ( $k$-NZF for short) of $M$ if $\forall e \in E(M), 0<|f(e)|<k$.

The matroid version of Conjecture 2.1.1 is as follows

Conjecture 1.2.1 If $M$ is a coloopless regular matroid such that $M \in E X\left(M\left(P_{10}\right), M^{*}\left(K_{5}\right)\right)$, then $M$ admits a 4 -NZF.

Applying the Four-Color theorem, and the duality between colorings and nowhere zero flows, a result by Walton and Welsh implies the following.

Theorem 1.2.2 (Walton and Welsh [48]) If $M \in E X\left(M\left(K_{3,3}\right), M^{*}\left(K_{5}\right)\right) \cap \mathcal{R}$ is a coloopless matroid, then $M$ admits a $4-N Z F$.

Proving a conjecture of Jensen and Toft [22], Lai, Li and Poon applied the Four-Color Theorem to prove the following Theorem 3.1.7, which is an approach to Conjecture 1.2.1.

Theorem 1.2.3 (Lai, Li and Poon, [27]) If $M \in E X\left(M\left(K_{5}\right), M^{*}\left(K_{5}\right)\right) \cap \mathcal{R}$ is a coloopless matroid, then $M$ admits a $4-N Z F$. 
The main objective of this chapter is to prove the following theorem, which generalizes Theorem 3.1.7, and is also an approach to Conjecture 1.2.1.

Theorem 1.2.4 If $M$ is a coloopless matroid such that $M \in E X\left(M\left(\left(P_{10}\right) \overline{3}\right), M^{*}\left(K_{5}\right)\right) \cap$ $\mathcal{R}$, then $M$ admits a $4-N Z F$.

\subsection{Odd-edge-connectivity of graphs}

It is evident that odd-edge-connectivity (see Definition 4.2.1) plays a more important role than edge-connectivity in the study of some flow and cycle cover related problems. In this article, some earlier results in those areas are extended from $\lambda$-edge-connected graphs to odd- $\lambda$-edge-connected graphs.

For graphs with large odd-edge-connectivity, small edge-cuts (of even size) may still exist. However, there are not many results or methods developed yet to deal with small even edge-cuts. For some integer flow problems and cycle cover problems, it is pointed out in $[36,21]$ that 2-edge-cut does not exit in any smallest counterexample (to some well-known flow conjectures and cycle cover conjectures). The 3-flow conjecture by Tutte [?] was originally proposed for odd-5-edge-connected graphs. By excluding 4-edge-cut, Kochol [23], with a sophisticated linear algebra approach, proved that 3-flow conjecture is equivalent for 5-edge-connected graphs.

In this chapter, we are to develop some general approaches to deal with small even cuts.

\subsubsection{Parity Subgraphs}

Definition 1.3.1 Let $H$ be a subgraph of a bridgeless graph $G=(V, E)$. $H$ is a parity subgraph of $G$ if for every vertex $v \in V(G), d_{G}(v) \equiv d_{H}(v)(\bmod 2)$.

It was proved by Tutte and Nash-Williams $[43,29]$ that every $2 k$-edge-connected graph 
contains at least $k$ edge-disjoint spanning trees, and proved by Itai and Rodeh [18] that every spanning tree of a graph $G$ contains a parity subgraph. The combination of these two theorems yields the following result as a direct corollary.

Theorem 1.3.2 Every $2 k$-edge-connected graph $G$ contains at least $k$ edge-disjoint parity subgraphs of $G$.

It is well-known that the search of parity subgraphs plays a central role in the proofs of some important theorems in integer flow areas. For example, the 4-flow theorem is proved by Jaeger [19] with following approach: The 4-edge-connectivity guarantees the existence of two edge-disjoint parity subgraphs (by Theorem 4.1.2) and therefore a 2-cycle cover of $G$. The 8-flow theorem was proved by Jaeger [19] with following similar approach: The 3-edge-connectivity guarantees the existence of three edge-disjoint parity subgraphs in $2 G$ and therefore, a 3 -cycle cover of $G$.

Theorem 4.1.2 is to be generalized in this article by relaxing the edge-connectivity to odd-edge-connectivity and therefore, solves an open problem proposed in [51, 12, 54].

We generalize the theorem and get the following

Theorem 1.3.3 Every odd-(2k+1)-edge-connected graph $G$ contains at least $k$ edgedisjoint parity subgraphs of $G$.

\subsubsection{Flow Index}

Circular flow, introduced in [16] as a real line extension of integer flow problem. The following is one of the many equivalent definitions for circular flows and the corresponding flow indices.

Definition 1.3.4 Let $\mathcal{D}$ be the set of all orientations of $G$, and $(A, B)$ be any edge-cut of $G$. The flow index of $G$ is defined by

$$
\phi(G)=\min _{D \in \mathcal{D}}\left\{\max _{(A, B)} \frac{\left|[A, B]_{D}\right|}{\left|[B, A]_{D}\right|}\right\}+1 .
$$


Theorem 1.3.5 (Galluccio and Goddyn, [15]) let $G$ be a 6-edge-connected graph, then the flow index $\phi(G)<4$.

We generalize the theorem above and get:

Theorem 1.3.6 Let $G$ be an odd-7-edge-connected graph, then $\phi(G)<4$.

\subsubsection{Nowhere-zero 3-flows}

The following is an approach of Jaeger's weak 3-flow conjecture [19]. Lai and Zhang proved the following theorem

Theorem 1.3.7 ([24]) Every $4\left\lceil\log _{2} n\right\rceil$-edge-connected multigraph with $n$ vertices admits a nowhere-zero 3-flow.

As a generalization, we prove

Theorem 1.3.8 Let $G$ be a multigraph with $n$ vertices. If its odd-edge-connectivity is more than $4\left\lceil\log _{2} n\right\rceil$, then $G$ admits a nowhere-zero 3-flow. 


\section{Chapter 2}

\section{4-NZF in almost $P_{10}$ minor free graphs}

\subsection{Introduction}

The concept of integer flow was introduced by Tutte as a generalization of map coloring problem. The following conjecture is one of the major open problems in graph theory.

Conjecture 2.1.1 (Tutte [45]) Every bridgeless graph without a Petersen minor admits a nowhere-zero 4 -flow.

For planar graphs, admitting a nowhere-zero 4-flow is equivalent to having a face 4coloring. Hence, by the 4-Color Theorem [?, 2, 3, ?], Conjecture 2.1.1 has been verified for all planar graphs. Furthermore, it was also announced that Conjecture 2.1.1 was verified for all cubic graphs [32, 33]. By Kuratowski Theorem, a graph is planar if and only if it contains neither $K_{5}$-minor nor $K_{3,3}$-minor. By applying the 4 -Color Theorem, Conjecture 2.1.1 was further verified for $K_{3,3}$-minor free graphs [48], $K_{5}$-minor free graphs [27], and $P_{10}^{-}$-minor free graphs [39]. Each of these families contains the family of all planar graphs and may not be necessarily cubic. Graphs $K_{5}, K_{3,3}, P_{10}$ and $P_{10}^{-}$are illustrated in 
the following figure.

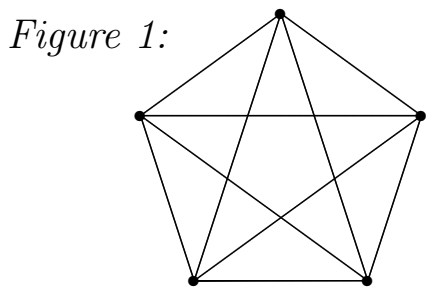

$K_{5}$
Figure 2:

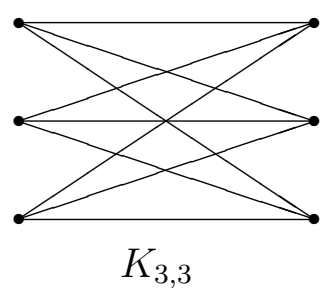

$K_{3,3}$

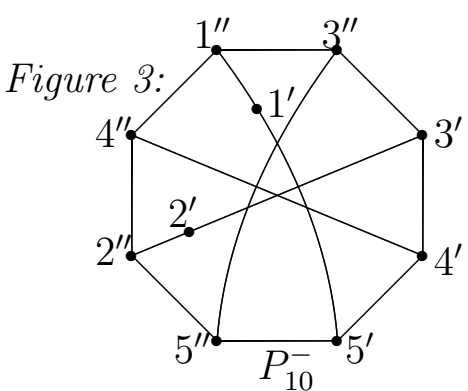

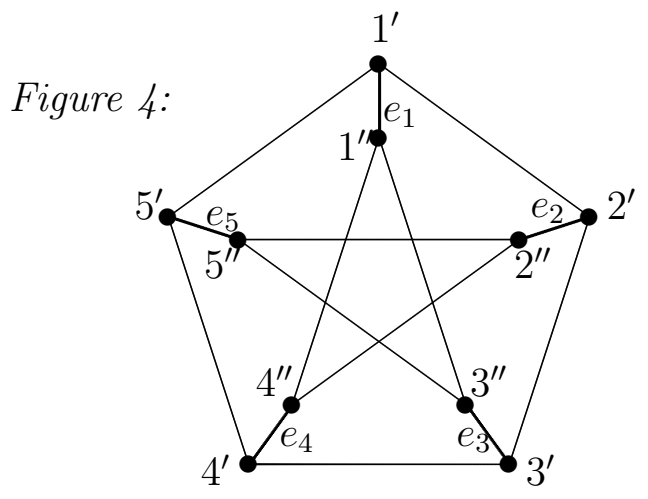

Petersen graph $P_{10}$

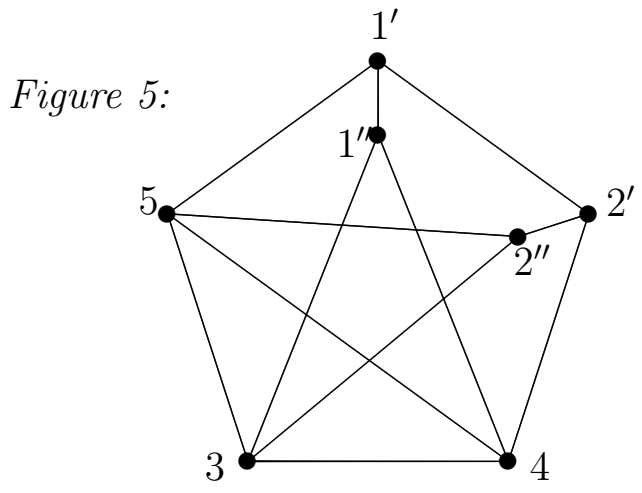

$\left(P_{10}\right)_{\overline{3}}=P_{10} /\left\{e_{3}, e_{4}, e_{5}\right\}$

Let $P_{10}$ be the Petersen graph with the exterior pentagon $1^{\prime} 2^{\prime} 3^{\prime} 4^{\prime} 5^{\prime} 1^{\prime}$, interior pentagon $1^{\prime \prime} 3^{\prime \prime} 5^{\prime \prime} 2^{\prime \prime} 4^{\prime \prime} 1^{\prime \prime}$ and a perfect matching $M=\left\{e_{i}=i^{\prime} i^{\prime \prime}: i=1,2,3,4,5\right\}$. Let $\left(P_{10}\right)_{\bar{\mu}}$ be the graph obtained from $P_{10}$ by contracting $F$, where $F \subseteq M$ and $|F|=\mu$.

Remark. It is not hard to see that if $M$ and $M^{\prime}$ are two perfect matchings of $P_{10}$, $F \subseteq M, F^{\prime} \subseteq M^{\prime}$ and $|F|=\left|F^{\prime}\right|$, then $P_{10} / F \cong P_{10} / F^{\prime}$. Hence $\left(P_{10}\right)_{\bar{\mu}}$ is well defined.

The following is our main theorem.

Theorem 2.1.2 Let $G$ be a bridgeless graph. If $G$ does not have a $\left(P_{10}\right)_{\overline{3}}$-minor, then $G$ admits a nowhere-zero 4-flow. 


\section{$2.2 \quad$ Notations and terminologies}

For terms that are not defined here, readers can refer to textbooks [5], [?], and [54] (for flows).

Let $G=(V, E)$ be a graph with vertex set $V$ and edge set $E$ and let $D$ be an orientation of $G$. For a vertex $v \in V(G)$, let $E^{+}(v)$ (or $E^{-}(v)$ ) be the set of all arcs of $D(G)$ with their tails (or heads, respectively) at the vertex $v$. $G$ is said to admit a nowhere-zero k-flow if there exists an ordered pair $(D, f)$, where $f: E(G) \rightarrow\{ \pm 1, \pm 2, \cdots \pm(k-1)\}$ such that

$$
\sum_{e \in E^{+}(v)} f(e)=\sum_{e \in E^{-}(v)} f(e)
$$

for every vertex $v \in V(G)$.

A graph $G$ is a 4-flow snark if it is bridgeless and does not admit a nowhere-zero 4-flow. Let $G$ and $H$ be two graphs. If $G$ contains a subgraph which is contractible to $H$, then $H$ is a minor of $G$ and we say $G$ contains an $H$-mimor. A 4-flow snark $G$ is minor-prime if every proper minor of $G$ is not a 4 -flow snark. With the definitions above, Conecjture 2.1.1 can be restated as follows.

Conjecture 2.2.1 The Petersen graph is the only minor-prime 4-flow snark.

Let $H$ be a minor of a connected graph $G$. Then there is an onto mapping $f$ : $V(G) \mapsto V(H)$ such that $f^{-1}(v)$ induces a connected subgraph $G\left[f^{-1}(v)\right]$ of $G$ for every $v \in V(H)$ and $H$ can be obtained from a spanning subgraph of $G$ by contracting the edges of $G\left[f^{-1}(v)\right]$ for all $v \in V(H)$. Here $f$ is called a minor-mapping and $f^{-1}(v)$ is called a $v$-domain of $f$.

A $k$-separator of a graph $G$ is an ordered triple $\left(H_{1}, H_{2} ; T\right)$ such that $H_{1} \cup H_{2}=G$ and $V\left(H_{1} \cap H_{2}\right)=T$, where $T$ is a vertex subset of $G$ and $|T|=k$. Sometimes we say $T$ is a $k$-separator if there is no confusion. A $k$-separator $\left(H_{1}, H_{2} ; T\right)$ of $G$ is trivial if one of $H_{1}$ and $H_{2}$, say $H_{1}$, is acyclic. $G$ is quasi $k$-connected if $G$ is 3-connected and every $t$-separator of $G$ with $t \leq k$ is trivial. 
Let $x$ be a vertex of $G$. The vertex $x$ separates $G$ into $q$ parts $H_{1}, \cdots, H_{q}$ if $G=$ $H_{1} \cup \cdots \cup H_{q}$ and $V\left(H_{i} \cap H_{j}\right)=\{x\}$ for every pair of $i \neq j$.

Let $X$ be a connected subgraph of $G$ and $Y_{1}, Y_{2}, Y_{3}, Y_{4}$ be four disjoint connected subgraphs of $G-V(X)$ and $X \cap N\left(Y_{i}\right) \neq \emptyset$ for $i=1,2,3,4$ where $N\left(Y_{i}\right)$ denotes the set of neighbors of $Y_{i}$. Let $J=\left\{Y_{1}, Y_{2}, Y_{3}, Y_{4}\right\}$. For each $2 \times 2$-partition $P=\{\{a, b\},\{c, d\}\}$ of $\{1,2,3,4\}, X$ is $P$-splittable if $X$ contains two disjoint paths $Q$ and $Q^{\prime}$ such that $Q$ joins $X \cap N\left(Y_{a}\right)$ and $X \cap N\left(Y_{b}\right), Q^{\prime}$ joins $X \cap N\left(Y_{c}\right)$ and $X \cap N\left(Y_{d}\right), i \in\{a, b, c, d\}$. An example of a $\{\{1,2\},\{3,4\}\}$-splittable subgraph is illustrated in Figure $6 . X$ is $k$-splittable with respect to $J$ if there are $k$ distinct $2 \times 2$ partitions $P_{1}, \cdots, P_{k}$ of $\{1,2,3,4\}$ such that $X$ is $P_{i}$-splittable for each $i=1, \cdots, k$. (Remark: $k \leq 3$ ) An example of a 2-splittable subgraph is illustrated in Figure 7.

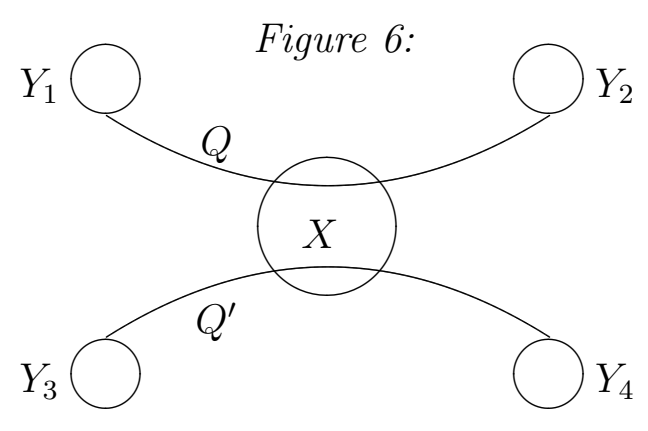

$X$ is $\{\{1,2\},\{3,4\}\}$-splittable

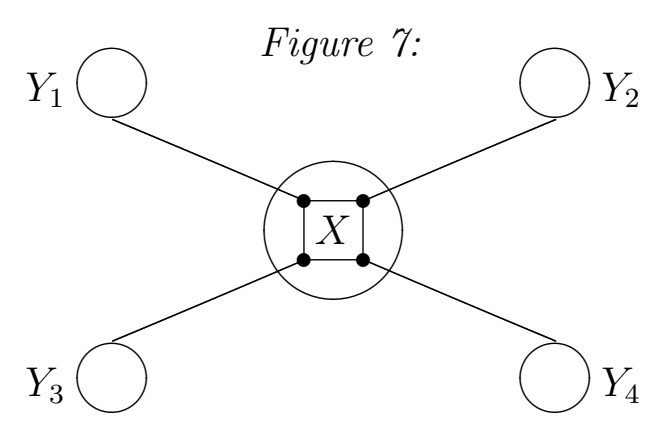

$X$ is 2-splittable

\subsection{Lemmas}

Lemma 2.3.1 (Catlin [9]) If $G$ is a minor-prime 4-flow snark, then the girth of $G$ is at least five.

Lemma 2.3.2 (Lai, Li and Poon [27]) If a bridgeless graph $G$ does not admit a nowherezero 4-flow, then $G$ has a $K_{5}$-minor.

Lemma 2.3.3 (Thomas and Thomson, Lemma 4.4 of [39]) If $G$ is a minor-prime 4-flow 
snark, then $G$ is quasi-4-connected (that is, every k-separator of $G$ is trivial for each $k \leq 3)$.

Obviously, Lemma 2.3.3 generalizes Lemma 2.3.1 and Theorem 3.7.15 of [54].

Proposition 2.3.4 Let $X$ be a connected subgraph of $G$ and $Y_{1}, Y_{2}, Y_{3}, Y_{4}$ be four disjoint connected subgraphs of $G-V(X)$ where $V(X) \cap N\left(Y_{i}\right) \neq \emptyset$ for $i=1,2,3,4$. Let $k$ be the greatest integer that $X$ is $k$-splittable with respect to $J=\left\{Y_{1}, Y_{2}, Y_{3}, Y_{4}\right\}$.

(i) If $k \leq 1$, say, $X$ is $\{\{1,2\},\{3,4\}\}$-splittable or 0 -splittable, then $X$ has a 1-separator $\left(H_{1}, H_{2} ;\{x\}\right)$ such that $V(X) \cap\left[N\left(Y_{1}\right) \cup N\left(Y_{2}\right)\right] \subseteq V\left(H_{1}\right)$ and $V(X) \cap\left[N\left(Y_{3}\right) \cup N\left(Y_{4}\right)\right] \subseteq$ $V\left(H_{2}\right)$;

(ii) If $k=0$, then there exists a cut vertex $x$ of $X$ that separates $X$ into four parts $H_{1}, H_{2}, H_{3}, H_{4}$ such that $V(X) \cap N\left(Y_{i}\right) \subseteq V\left(H_{i}\right)$ for each $i$.

Proof. (i) Let $G_{1}$ be the graph induced by $X \cup Y_{1} \cup Y_{2} \cup Y_{3} \cup Y_{4}$. Let $G_{2}$ be the graph obtained from $G_{1}$ by contracting each $Y_{i}$ into a single vertex $y_{i}$ for $i=1,2,3,4$, and deleting all edges between $y_{i}$ and $y_{j}$ for all $\{i, j\} \subset\{1,2,3,4\}$. Note that $G_{2}$ is connected since $V(X) \cap N\left(Y_{i}\right) \neq \emptyset$ for $i=1,2,3,4$.

Since $X$ is neither $\{\{1,3\},\{2,4\}\}$-splittable nor $\{\{1,4\},\{2,3\}\}$-splittable, it is impossible that there is a pair of disjoint paths joining $\left\{y_{1}, y_{2}\right\}$ and $\left\{y_{3}, y_{4}\right\}$. By Menger theorem, there is a cut vertex $x \in V\left(G_{2}\right)$ that separates $\left\{y_{1}, y_{2}\right\}$ and $\left\{y_{3}, y_{4}\right\}$. It is obvious that $x \in V(X)$. That is, $X$ has a 1-separator $\left(H_{1}, H_{2} ; x\right)$ that $N_{G_{2}}\left(y_{1}\right) \cup N_{G_{2}}\left(y_{2}\right) \subseteq V\left(H_{1}\right)$ and $N_{G_{2}}\left(y_{3}\right) \cup N_{G_{2}}\left(y_{4}\right) \subseteq V\left(H_{2}\right)$.

(ii) Continue from (i). Assume that there is a path $P_{1}$ joining $y_{1}$ and $y_{2}$ in the graph $G_{2}-\{x\}$ (without passing through $x$ ). Note that $x$ is a cut vertex that separates $\left\{y_{1}, y_{2}\right\}$ and $\left\{y_{3}, y_{4}\right\}$. Thus, this path $P_{1}$ is contained in the induced subgraph $G_{2}\left[V\left(H_{1}-\right.\right.$ $\left.x) \cup\left\{y_{1}, y_{2}\right\}\right]$ and there is another path $P_{2}$ joining $y_{3}$ and $y_{4}$ in the induced subgraph $G_{2}\left[H_{2} \cup\left\{y_{3}, y_{4}\right\}\right.$ since $H_{2}$ is connected. This contradicts that $X$ is 0 -splittable. So every path from $y_{1}$ to $y_{2}$ must go through $x$. Symmetrically, every path from $y_{3}$ to $y_{4}$ must go 
through $x$ as well. That implies each component of $X-x$ is adjacent to at most one of $\left\{y_{1}, y_{2}, y_{3}, y_{4}\right\}$.

\subsection{Proof of the main theorem}

Let $G$ be a minor-prime 4-flow snark. By Lemma 2.3.3, $G$ is quasi 4-connected. By Lemma 2.3.2, $K_{5}$ is a minor of $G$. Let $V\left(K_{5}\right)=\left\{v_{1}, v_{2}, v_{3}, v_{4}, v_{5}\right\}$, and $f: V(G) \mapsto V\left(K_{5}\right)$ be a minor-mapping.

If $G$ does not contain a $\left.\left(P_{10}\right)\right)_{4}$-minor, then $v_{a}$-domain $f^{-1}\left(v_{a}\right)$ is at most 0 -splittable with respect to $\left\{f^{-1}\left(v_{i_{j}}\right): j=1,2,3,4\right\}$ for every $\left\{a, i_{1}, i_{2}, i_{3}, i_{4}\right\}=\{1,2,3,4,5\}$. By Lemma 2.3.4(ii), each $f^{-1}\left(v_{a}\right)$ has a cut vertex $v_{a}^{*}$ that separates $N\left(f^{-1}\left(v_{i_{j}}\right)\right)$ for $j=$ $1,2,3,4$. Hence $\left\{v_{i}^{*}, v_{j}^{*}\right\}$ is a 2 -separator of $G$. Since $G \neq K_{5}$, there exist $\{i, j\} \subseteq$ $\{1,2,3,4,5\}$ such that $\left\{v_{i}^{*}, v_{j}^{*}\right\}$ is a non-trivial 2-separator. This contradicts the fact that $G$ is quasi 4 -connected. Hence $G$ contains $\left(P_{10}\right)_{\overline{4}}$ as a minor.

Let $f: V(G) \rightarrow\left(P_{10}\right)_{\overline{4}}$ be a minor mapping where the vertex set of $\left(P_{10}\right)_{\overline{4}}$ is $\left\{v_{1^{\prime}}, v_{1^{\prime \prime}}\right.$, $\left.v_{2}, v_{3}, v_{4}, v_{5}\right\}$, the contraction of the edge $v_{1^{\prime}} v_{1^{\prime \prime}}$ yields a $K_{5}, v_{1^{\prime}}$ is adjacent to $v_{2}$ and $v_{5}$, and $v_{1^{\prime \prime}}$ is adjacent to $v_{3}$ and $v_{4}$. Let $U_{i}=f^{-1}\left(v_{i}\right)$ for $i \in\left\{1^{\prime}, 1^{\prime \prime}, 2,3,4,5\right\}$ (see the following figure). Denote $U_{1}=U_{1^{\prime}} \cup U_{1^{\prime \prime}}$ and choose a minor-mapping $f$ such that $\left|U_{1}\right|$ is as small as possible. Now assume that $G$ does not contain a $\left(P_{10}\right) \overline{3}$-minor.

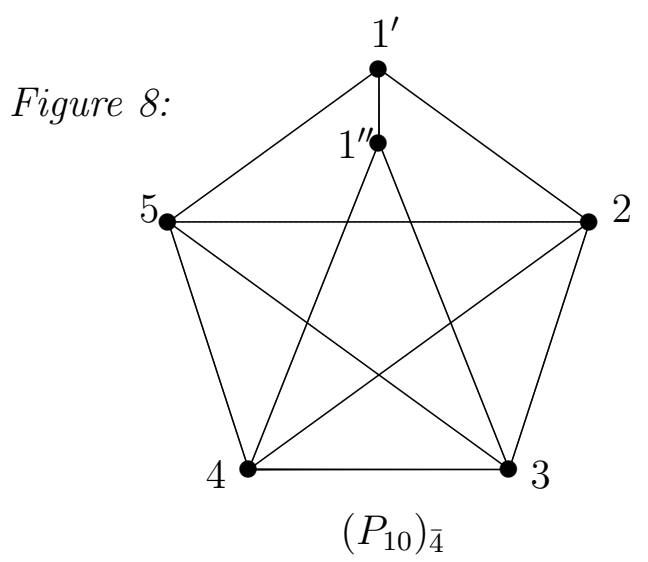


Claim $1\left|U_{1^{\prime}}\right|=\left|U_{1^{\prime \prime}}\right|=1$.

Proof. It is clear that $\left|U_{1^{\prime}}\right| \geq 1$ and $\left|U_{1^{\prime \prime}}\right| \geq 1$.

Let $e=u_{1^{\prime}} u_{1^{\prime \prime}}$ be an edge between $U_{1^{\prime}}$ and $U_{1^{\prime \prime}}$ where $u_{1^{\prime}} \in U_{1^{\prime}}, u_{1^{\prime \prime}} \in U_{1^{\prime \prime}}$. Since each of $U_{1^{\prime}}$ and $U_{1^{\prime \prime}}$ is connected, there are spanning trees $T_{1}$ and $T_{2}$ of $U_{1^{\prime}}$ and $U_{1^{\prime \prime}}$, respectively. Let $T=T_{1} \cup T_{2} \cup\{e\}$. $T$ is a spanning tree of $U_{1}$.

Assume there exist $w_{2} \in N\left(U_{2}\right) \cap U_{1^{\prime}}$ and $w_{5} \in N\left(U_{5}\right) \cap U_{1^{\prime}}$ such that $w_{2} \neq w_{5}$. Since $T_{1}$ is a spanning tree of $U_{1^{\prime}}$, there is a unique path $P_{2}$ from $u_{1^{\prime}}$ to $w_{2}$ in $T_{1}$, and a path $P_{5}$ from $u_{1^{\prime}}$ to $w_{5}$ in $T_{1}$. Without loss of generality, we may assume $P_{2}$ is no shorter than $P_{5}$. Since $w_{2} \neq w_{5}, P_{5}$ does not contain $w_{2}$. Let $C_{2}$ be the set of vertices of the component of $T_{1} \backslash P_{5}$ that contains $w_{2}$. Now we define a new minor mapping $f_{1}$ by $f_{1}^{-1}\left(v_{i}\right)=f^{-1}\left(v_{i}\right)$ for $i=1^{\prime \prime}, 3,4,5, f_{1}^{-1}\left(v_{1^{\prime}}\right)=f^{-1}\left(v_{1^{\prime}}\right) \backslash C_{2}$ and $f_{1}^{-1}\left(v_{2}\right)=f^{-1}\left(v_{2}\right) \cup C_{2}$. We call this operation moving $w_{2}$ from $U_{1^{\prime}}$ to $U_{2}$.

$$
\left|f_{1}^{-1}\left(v_{1^{\prime}}\right) \cup f_{1}^{-1}\left(v_{1^{\prime \prime}}\right)\right|=\left|f^{-1}\left(v_{1^{\prime}}\right) \cup f^{-1}\left(v_{1^{\prime \prime}}\right)\right|-\left|C_{2}\right|<\left|f^{-1}\left(v_{1^{\prime}}\right) \cup f^{-1}\left(v_{1^{\prime \prime}}\right)\right|
$$

That contradicts the choice of $f$. So we have $N\left(U_{2}\right) \cap U_{1^{\prime}}=N\left(U_{5}\right) \cap U_{1^{\prime}}=\{u\}$ for some $u$. Similarly, $N\left(U_{3}\right) \cap U_{1^{\prime \prime}}=N\left(U_{4}\right) \cap U_{1^{\prime \prime}}=\{v\}$ for some $v$.

Since $G$ is quasi 4-connected, if $\{u, v\}$ is a 2-separator, then $\left|U_{1}\right|=2$. If $\left|U_{1}\right| \geq 3$, then $\{u, v\}$ is not a 2-separator and there exists $w \in U_{1} \backslash\{u, v\}$ such that $w \in N\left(U_{i}\right)$ for some $i=2,3,4,5$. Without loss of generality, we can assume $w \in N\left(U_{2}\right)$.

Since $N\left(U_{2}\right) \cap U_{1^{\prime}}=\{u\}, w \notin U_{1^{\prime}}$. If the path $P$ from $u$ to $v$ in $T$ passes through $w$, then we can move $w$ from $U_{1^{\prime \prime}}$ to $U_{1^{\prime}}$, that contradicts $w \notin U_{1^{\prime}}$. If $P$ does not pass through $w$, then we can move $w$ from $U_{1^{\prime \prime}}$ to $U_{2}$, that contradicts the choice of $f$.

From Claim 1, we can let $U_{1^{\prime}}=\left\{u_{1^{\prime}}\right\}$ and $U_{1^{\prime \prime}}=\left\{u_{1^{\prime \prime}}\right\}$.

Claim $2 U_{2}$ is at most 1-splittable with respect to $J=\left\{1^{\prime}, 3,4,5\right\}$ with a possible partition $\left\{\left\{1^{\prime}, 5\right\},\{3,4\}\right\}$.

Proof. $U_{2}$ is neither $\left\{\left\{1^{\prime}, 3\right\},\{4,5\}\right\}$-splittable nor $\left\{\left\{1^{\prime}, 4\right\},\{3,5\}\right\}$-splittable. Otherwise we can have $\left(P_{10}\right)_{\overline{3}}$-minors illustrated in Figures 5 and 6 , respectively. 

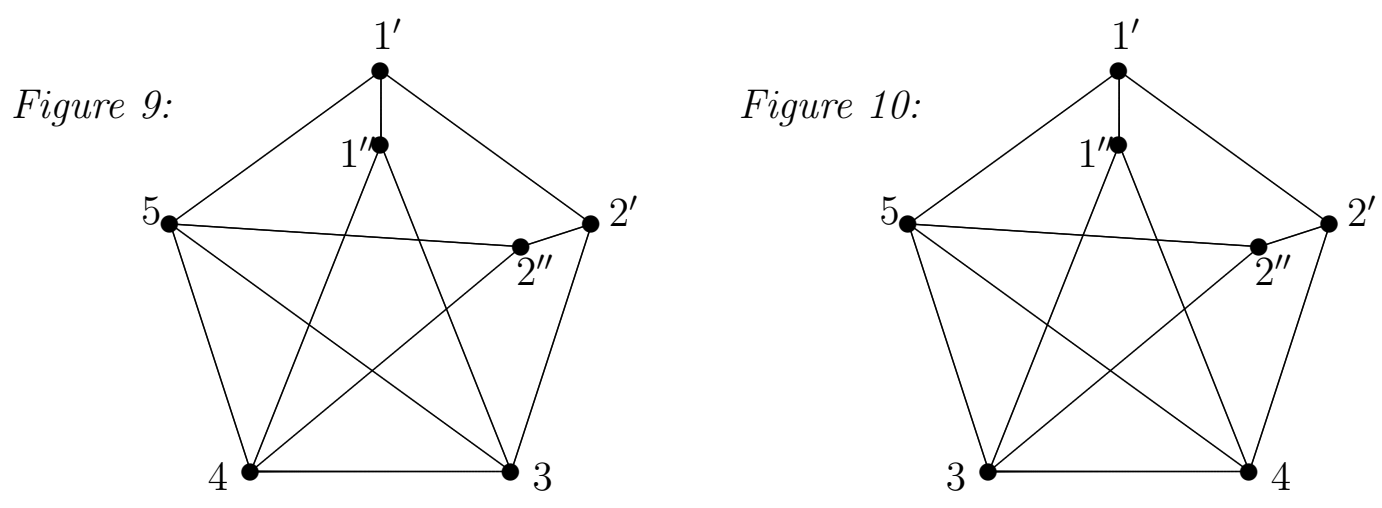

$U_{2}$ is $\left\{\left\{1^{\prime}, 3\right\},\{4,5\}\right\}$-splittable

$U_{2}$ is $\left\{\left\{1^{\prime}, 4\right\},\{3,5\}\right\}$-splittable

By Proposition 2.3.4(i), $U_{2}$ has a 1-separator $\left(A_{2}, B_{2} ; x_{2}\right)$ such that $\left[N\left(U_{1^{\prime}}\right) \cup N\left(U_{5}\right)\right] \cap$ $U_{2} \subseteq A_{2}$ and $\left[N\left(U_{3}\right) \cup N\left(U_{4}\right)\right] \cap U_{2} \subseteq B_{2}$, as we can see in Figure 11 .

Symmetrically, we have the following conclusions (as shown in Figure 12):

(i) $U_{5}$ is at most 1 -splittable with respect to $J=\left\{1^{\prime}, 2,3,4\right\}$ with the only possible $(2 \times 2)$-partition $\left\{\left\{1^{\prime}, 2\right\},\{3,4\}\right\}$ and it has a 1-separator $\left(A_{5}, B_{5} ; x_{5}\right)$ such that $\left[N\left(U_{1^{\prime}}\right) \cup\right.$ $\left.N\left(U_{2}\right)\right] \cap U_{5} \subseteq A_{5}$, and $\left[N\left(U_{3}\right) \cup N\left(U_{4}\right)\right] \cap U_{5} \subseteq B_{5}$.

(ii) $U_{3}$ is at most 1 -splittable with respect to $J=\left\{1^{\prime \prime}, 2,4,5\right\}$ with the only possible $(2 \times 2)$-partition $\left\{\left\{1^{\prime \prime}, 4\right\},\{2,5\}\right\}$ and it has a 1-separator $\left(A_{3}, B_{3} ; x_{3}\right)$ such that $\left[N\left(U_{1^{\prime \prime}}\right) \cup\right.$ $\left.N\left(U_{4}\right)\right] \cap U_{3} \subseteq A_{3}$, and $\left[N\left(U_{2}\right) \cup N\left(U_{5}\right)\right] \cap U_{3} \subseteq B_{3}$.

(iii) $U_{4}$ is at most 1 -splittable with respect to $J=\left\{1^{\prime \prime}, 2,3,5\right\}$ with the only possible $(2 \times 2)$-partition $\left\{\left\{1^{\prime \prime}, 3\right\},\{2,5\}\right\}$ and it has a 1-separator $\left(A_{4}, B_{4} ; x_{4}\right)$ such that $\left[N\left(U_{1^{\prime \prime}}\right) \cup\right.$ $\left.N\left(U_{3}\right)\right] \cap U_{4} \subseteq A_{4}$, and $\left[N\left(U_{2}\right) \cup N\left(U_{5}\right)\right] \cap U_{4} \subseteq B_{4}$. 


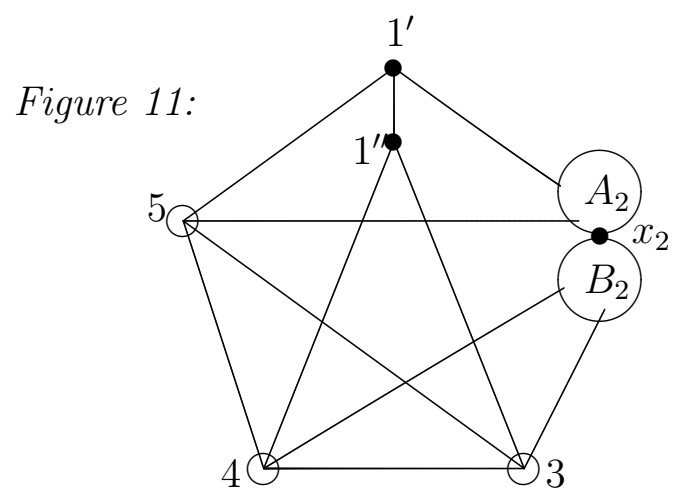

$U_{2}$ is at most 1-splittable w.r.t. $J=\left\{1^{\prime}, 3,4,5\right\}$

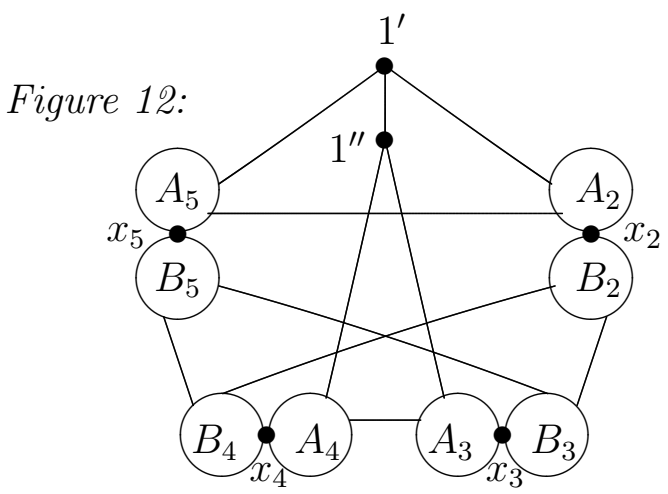

Each $U_{i}$ is at most 1-splittable for $i=2,3,4,5$

Claim $3\left\{N\left(u_{1^{\prime \prime}}\right) \cap A_{2}-\left\{x_{2}\right\}\right\} \cup\left\{N\left(u_{1^{\prime \prime}}\right) \cap A_{5}-\left\{x_{5}\right\}\right\} \neq \emptyset$.

Proof. Otherwise, $T=\left\{u_{1^{\prime}}, x_{2}, x_{5}\right\}$ is a non-trivial 3-separator of $G$ that separates $G$ with $A_{2} \cup A_{5} \cup U_{1^{\prime}}$ as one part. By Lemma 2.3.3, $G$ is quasi 4-connected, therefore, $A_{2} \cup A_{5} \cup U_{1^{\prime}}$ is trivial, but it is not acyclic.

Similarly, $\left\{N\left(u_{1^{\prime}}\right) \cap A_{3}-\left\{x_{3}\right\}\right\} \cup\left\{N\left(u_{1^{\prime}}\right) \cap A_{4}-\left\{x_{4}\right\}\right\} \neq \emptyset$.

Without loss of generality, we assume that

$$
\left\{N\left(u_{1^{\prime}}\right) \cap A_{3}-\left\{x_{3}\right\}\right\} \neq \emptyset, \quad\left\{N\left(u_{1^{\prime \prime}}\right) \cap A_{2}-\left\{x_{2}\right\}\right\} \neq \emptyset .
$$

Claim $4 U_{2}$ is not $\left\{\left\{1^{\prime \prime}, 5\right\},\{3,4\}\right\}$-splittable.

Proof. Otherwise $G$ has a $\left(P_{10}\right) \overline{3}$-minor as in Figure 13 (note that the edge between $U_{1^{\prime}}$ and $U_{3}$ is given by $(2.1)$.

Symmetrically, $U_{5}$ is not $\left\{\left\{1^{\prime \prime}, 2\right\},\{3,4\}\right\}$-splittable.

Claim $5 U_{2}$ is at most 0-splittable with respect to $J=\left\{1^{\prime}, 3,4,5\right\}$. 
Proof. By way of contradiction, assume $U_{2}$ is not 0 -splittable with respect to $J=$ $\left\{1^{\prime}, 3,4,5\right\}$. By Claim $2, U_{2}$ is $\left\{\left\{1^{\prime}, 5\right\},\{3,4\}\right\}$-splittable.

Let $\left\{P_{1^{\prime}, 5}, P_{3,4}\right\}$ be a pair of vertex disjoint paths in $U_{2}$ that $P_{i j}$ joins $N\left(U_{i}\right) \cap U_{2}$ and $N\left(U_{j}\right) \cap U_{2}$ for $i, j \in\left\{1^{\prime}, 3,4,5\right\}$.

It is obvious that $P_{3,4}$ must contain the cut vertex $x_{2}$ for otherwise $A_{2}$ contains a path joining $N\left(u_{1^{\prime \prime}}\right)$ and $N\left(U_{5}\right)$. This contradicts Claim 4. Therefore, $N\left(U_{1^{\prime}}\right) \cap\left(A_{2}-x_{2}\right) \neq \emptyset$, $N\left(U_{5}\right) \cap\left(A_{2}-x_{2}\right) \neq \emptyset$ and both of them are contained in the same component of $A_{2}-x_{2}$, called $C_{2}$, while $N\left(U_{1^{\prime \prime}}\right) \cap\left(A_{2}-x_{2}\right)$ is contained in another component of $A_{2}-x_{2}$.

Symmetrically, $A_{5}-x_{5}$ has a component $C_{5}$ that contains $N\left(U_{1^{\prime}}\right) \cap\left(A_{5}-x_{5}\right)$ and $N\left(U_{2}\right) \cap\left(A_{5}-x_{5}\right)$ and is disjoint with $N\left(U_{1^{\prime \prime}}\right)$.

Here we have obtained a 3 -separator $\left(H_{1}, H_{2} ; T\right)$ with $T=\left\{u_{1^{\prime}}, x_{2}, x_{5}\right\}$ as the cut and $H_{1}=C_{2} \cup C_{5} \cup\left\{u_{1^{\prime}}, x_{2}, x_{5}\right\}$. Note that neither $H_{1}$ nor $H_{2}$ is trivial. This contradicts Lemma 2.3.3.

Similarly, $U_{3}$ is at most 0 -splittable with respect to $J=\left\{1^{\prime \prime}, 3,4,5\right\}$.
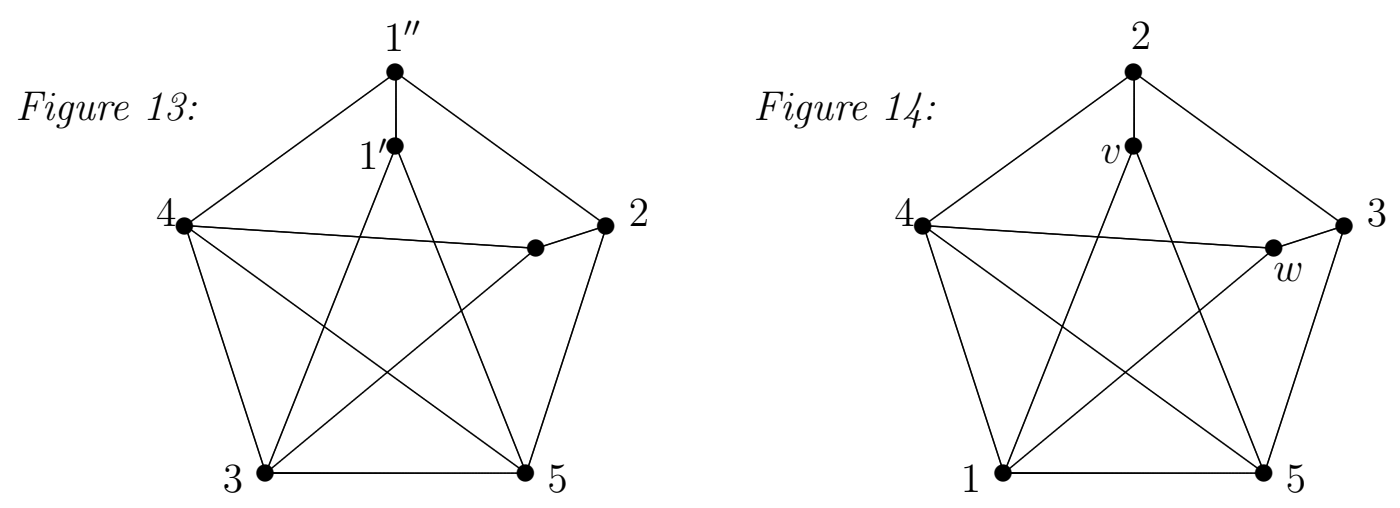

when $U_{2}$ is $\left\{\left\{1^{\prime \prime}, 5\right\},\{3,4\}\right\}$-splittable

\section{Final Step:}

By Claim 5 and Proposition 2.3.4(ii), $x_{2}$ separates $U_{2}$ into four parts $U_{2}\left(1^{\prime}\right), U_{2}(5)$, $U_{2}(4)$ and $U_{2}(3)$ such that $N\left(U_{i}\right) \cap U_{2} \subseteq U_{2}(i)$ for $i \in\left\{1^{\prime}, 3,4,5\right\}$. 
By Claim 3, $N\left(u_{1^{\prime \prime}}\right) \cap A_{2}-\left\{x_{2}\right\} \neq \emptyset$. Assume that $N\left(u_{1^{\prime \prime}}\right) \cap A_{2}-\left\{x_{2}\right\} \subseteq U_{2}\left(1^{\prime}\right)-x_{2}$. Then $\left\{u_{1^{\prime}}, u_{1^{\prime \prime}}, x_{2}\right\}$ is a 3 -separator of $G$ with $U_{2}\left(1^{\prime}\right) \cup U_{1}$ as a part. Both parts of $G$ separated by $\left\{u_{1^{\prime}}, u_{1^{\prime \prime}}, x_{2}\right\}$ contain cycles. This contradicts Lemma 2.3.3. So, there exists a vertex $v \in U_{2}(5) \cap N\left(u_{1^{\prime \prime}}\right)-\left\{x_{2}\right\}$ since $A_{2}=U_{2}\left(1^{\prime}\right) \cup U_{2}(5)$.

Similarly, there is a vertex $w \in N\left(u_{1^{\prime}}\right) \cap A_{3}-\left\{x_{3}\right\}$, which deduces $w \in U_{3}(4)$. Now we have a $\left(P_{10}\right) \overline{3}$-minor as in Figure 14 . 


\section{Chapter 3}

\section{4-NZF in regular matroids}

\subsection{Introduction}

We shall assume familiarity with graph theory and matroid theory. For terms that are not defined in this note, see Bondy and Murty [5] for graphs, and Oxley [30] or Welsh [50] for matroids.

In this chapter, $\mathbf{Z}, \mathbf{Z}^{+}$and $\mathbf{Z}_{n}$ denote the additive group of the integers, the set of all positive integers, and the cyclic group of order $n$, respectively, and $\mathcal{R}$ denotes the family of all regular matroids. As in [30], the set of all circuits of a matroid $M$ is denoted by $\mathcal{C}(M)$. We further denote the set of all cycles of a matroid $M$ by $\mathcal{C}_{0}(M)$. Note that as we allow empty unions, the empty set is also a cycle (in both graphs and matroids). For matroids $N_{1}, N_{2}, \cdots, N_{k}$, let $E X\left(N_{1}, N_{2}, \cdots, N_{k}\right)$ denote the collection of matroids such that a matroid $M \in E X\left(N_{1}, N_{2}, \cdots, N_{k}\right)$ if and only if $M$ does not have a minor isomorphic to any one in $\left\{N_{1}, N_{2}, \cdots, N_{k}\right\}$. The Fano matroid $F_{7}$ is the vector matroid over $\mathrm{GF}(2)$ of the following matrix $A$ :

$$
A=\left[\begin{array}{lllllll}
1 & 0 & 0 & 1 & 1 & 0 & 1 \\
0 & 1 & 0 & 1 & 0 & 1 & 1 \\
0 & 0 & 1 & 0 & 1 & 1 & 1
\end{array}\right]
$$


Flow was initially defined for graphs. For a discussion on flow and flow conjectures, see Jaeger [20] or Zhang [54]. The definition of flow has a natural extension to regular matroids. Let $M$ be a regular matroid and $D_{M}$ be its incidence matrix of circuits against elements. An orientation $\left(w\left(D_{M}\right), w\left(D_{M^{*}}\right)\right)$ is an assignment of,+- signs to the " 1 " entries of $D_{M}$ and $D_{M^{*}}$, respectively, so that the resulting matrices $w\left(D_{M}\right)$ and $w\left(D_{M^{*}}\right)$ satisfy

$$
w\left(D_{M}\right) w\left(D_{M^{*}}\right)^{T}=0 .
$$

Let $A$ be an abelian group. For an element $a \in A$, and for integers $+1,-1,0$, we adopt the convention to write $(+1) \cdot a=a,(-1) \cdot a=-a$ and $0 \cdot a=0$. Let $F^{*}(M, A)=$ $\{f: E(M) \mapsto A \backslash\{0\}\}$ denote the set of all functions from $E(M)$ into $A \backslash\{0\}$. A map $f \in F^{*}(M, A)$ can be viewed as an $|E(M)|$-dimensional column vector. For a regular matroid $M$ with an orientation $\left(w\left(D_{M}\right), w\left(D_{M^{*}}\right)\right)$, a map $f \in F^{*}(M, A)$ satisfying

$$
w\left(D_{M^{*}}\right) \cdot f=0
$$

is a nowhere zero $A$-flow ( $A$-NZF for short) of $M$. When $A=\mathbf{Z}$, a $\mathbf{Z}$-NZF $f$ of $M$ is called a nowhere zero $k$-flow ( $k$-NZF for short) of $M$ if $\forall e \in E(M), 0<|f(e)|<k$.

For positive integers $k$ and $m$, an $m$-cycle $k$-cover of a matroid $M$ is a family of cycles $C_{1}, C_{2}, \cdots, C_{m}$ of $M$ such that every element of $E(M)$ lies in exactly $k$ members of these $C_{i}$ 's. It has been observed that a graph $G$ admits a 4 -NZF if and only if $G$ has a 3-cycle 2-cover (for example, see Zhang [54]). The following fact will be needed, a formal proof of it can be found in [27].

Proposition 3.1.1 (Proposition 1.1 of [27]) For a matroid $M \in \mathcal{R}, M$ admits a 4 -NZF if and only if $M$ has a 3-cycle 2-cover.

Let $P_{10}$ be the Petersen graph. Tutte proposed the famous 4 -flow conjecture as follows.

Conjecture 3.1.2 (Tutte [45] and [46], Matthews [28]) Let $G$ be a 2-edge-connected graph. If $G$ does not have a $P_{10}$-minor, then $G$ admits a 4-NZF. 
The matroid version of the conjecture is as follows

Conjecture 3.1.3 If $M$ is a coloopless regular matroid such that $M \in E X\left(M\left(P_{10}\right), M^{*}\left(K_{5}\right)\right)$, then $M$ admits a 4 -NZF.

The Four-Color theorem can be stated in terms of nowhere zero flows as follows.

Theorem 3.1.4 (Appel and Haken [1], Appel, Haken and Hoch [2], Robertson, Sanders, Seymour and Thomas [31]) Every 2-edge-connected planar graph admits a 4-NZF.

Recently Robertson et. al. prove Conjecture 3.1.2 for cubic graphs.

Theorem 3.1.5 (Robertson, Sanders, Seymour and Thomas, [33]) Every 2-edge-connected cubic graph without a minor isomorphic to the Petersen graph admits a 4-NZF.

Applying the Four-Color theorem, and the duality between colorings and nowhere zero flows, a result by Walton and Welsh implies the following.

Theorem 3.1.6 (Walton and Welsh [48]) If $M \in E X\left(M\left(K_{3,3}\right), M^{*}\left(K_{5}\right)\right) \cap \mathcal{R}$ is a coloopless matroid, then $M$ admits a 4-NZF.

Proving a conjecture of Jensen and Toft [22], Lai, Li and Poon applied the Four-Color Theorem to prove the following Theorem 3.1.7, which is an approach to Conjecture 3.1.3.

Theorem 3.1.7 (Lai, Li and Poon, [27]) If $M \in E X\left(M\left(K_{5}\right), M^{*}\left(K_{5}\right)\right) \cap \mathcal{R}$ is a coloopless matroid, then $M$ admits a 4-NZF.

The main objective of this chapter is to prove the following theorem, which generalizes Theorem 3.1.7, and is also an approach to Conjecture 3.1.3. 
Theorem 3.1.8 If $M$ is a coloopless matroid such that $M \in E X\left(M\left(\left(P_{10}\right) \overline{3}\right), M^{*}\left(K_{5}\right)\right) \cap$ $\mathcal{R}$, then $M$ admits a $4-N Z F$.

The definition of flow has no natural extension to binary matroids whereas cycle cover is defined for general matroids. In view of Proposition 3.1.1 and the excludedminor characterization of regular matroids, Theorem 3.1 .8 is equivalent to saying that if a coloopless binary matroid $M \in E X\left(F_{7}, F_{7}^{*}, M\left(\left(P_{10}\right) \overline{3}\right), M^{*}\left(K_{5}\right)\right)$, then $M$ has a 3 -cycle 2-cover. In Section 3 we will show that this result can be extended in the following form.

Corollary 3.1.9 Let $M$ be a coloopless binary matroid. If $M \in E X\left(F_{7}^{*}, M\left(\left(P_{10}\right) \overline{3}\right), M^{*}\left(K_{5}\right)\right)$, $M$ has a 3-cycle 2-cover.

As the matroid $F_{7}^{*}$ does not have a 3-cycle 2-cover (to be shown in Section 3), Corollary 3.1.9 does not hold if $F_{7}^{*}$ is not excluded.

In Section 2, we extract a decomposition theorem for regular matroids without $M\left(K_{5}\right)$ or $M^{*}\left(K_{5}\right)$ minors from the well known decomposition theorems of Seymour [35] and Wagner [47]. In Section 3, this theorem will be employed to prove Theorem 3.1.8 and Corollary 3.1.9.

\subsection{Decomposition of Regular Matroids in $\operatorname{EX}\left(M\left(K_{5}\right), M^{*}\left(K_{5}\right)\right)$}

In this paper, we use $\triangle$ to denote both a set operator and a matroid operator. Given two sets $X$ and $Y$, the symmetric difference of $X$ and $Y$ is defined as

$$
X \triangle Y=(X \cup Y)-(X \cap Y) .
$$

Definition 3.2.1 Suppose that $M_{1}, M_{2}$ are binary matroids on $E_{1}$ and $E_{2}$, respectively. We follow Seymour [35] and define a new binary matroid $M_{1} \triangle M_{2}$ to be the matroid with ground set equal to $E_{1} \triangle E_{2}$ and with its set of cycles equal to

$$
\left\{C_{1} \triangle C_{2} \subseteq E_{1} \triangle E_{2}: C_{i} \text { is a cycle of } M_{i}, i=1,2\right\} \text {. }
$$


Definition 3.2.2 Three special cases of this operation are introduced by Seymour ([35] and [37]) as follows.

(i) If $E_{1} \cap E_{2}=\emptyset$ and $\left|E_{1}\right|,\left|E_{2}\right|<\left|E_{1} \triangle E_{2}\right|, M_{1} \triangle M_{2}$ is a 1-sum of $M_{1}$ and $M_{2}$.

(ii) If $\left|E_{1} \cap E_{2}\right|=1$ and $E_{1} \cap E_{2}=\{z\}$, say, and $z$ is not a loop or coloop of $M_{1}$ or $M_{2}$, and $\left|E_{1}\right|,\left|E_{2}\right|<\left|E_{1} \triangle E_{2}\right|, M_{1} \triangle M_{2}$ is a 2-sum of $M_{1}$ and $M_{2}$.

(iii) If $\left|E_{1} \cap E_{2}\right|=3$ and $E_{1} \cap E_{2}=Z$, say, and $Z$ is a circuit of $M_{1}$ and $M_{2}$, and $Z$ includes no cocircuit of either $M_{1}$ or $M_{2}$, and $\left|E_{1}\right|,\left|E_{2}\right|<\left|E_{1} \triangle E_{2}\right|, M_{1} \triangle M_{2}$ is a 3-sum of $M_{1}$ and $M_{2}$.

For $i=1,2,3$, an $i$-sum of $M_{1}, M_{2}$ is denoted as $M_{1} \oplus_{i} M_{2}$. The 1 -sum $M_{1} \oplus_{1} M_{2}$ is also written as $M_{1} \oplus M_{2}$. Let $R_{10}$ denote the vector matroid of the following matrix over $G F(2)$ :

$$
R_{10}=\left[\begin{array}{llllllllll}
1 & 0 & 0 & 0 & 0 & 1 & 1 & 0 & 0 & 1 \\
0 & 1 & 0 & 0 & 0 & 1 & 1 & 1 & 0 & 0 \\
0 & 0 & 1 & 0 & 0 & 0 & 1 & 1 & 1 & 0 \\
0 & 0 & 0 & 1 & 0 & 0 & 0 & 1 & 1 & 1 \\
0 & 0 & 0 & 0 & 1 & 1 & 0 & 0 & 1 & 1
\end{array}\right]
$$

It is known that $R_{10}^{*}$ is isomorphic to $R_{10}$. Based on the notion of matroid sums, Seymour proved the following decomposition theorem for regular matroids.

Theorem 3.2.3 (Seymour [35]) Let $M$ be a regular matroid. One of the following must hold.

(i) $M$ is graphic.

(ii) $M$ is cographic.

(iii) $M \cong R_{10}$.

(iv) For some $i \in\{1,2,3\}, M=M_{1} \oplus_{i} M_{2}$ is the $i$-sum of two matroids $M_{1}$ and $M_{2}$, each of which is isomorphic to a proper minor of $M$.

If a matroid $M$ is isomorphic to the cycle matroid of a planar graph, then $M$ is called a planar matroid. Thus a matroid $M$ is planar if and only if $M^{*}$ is planar. Let $H_{8}$ 
denote the graph depicted in the figure below.

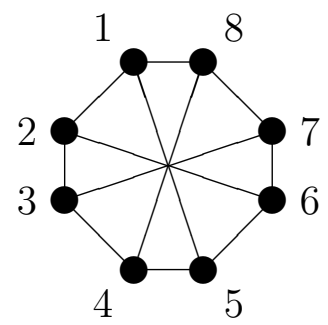

Figure 1: The graph $H_{8}$

Wagner's original statement of his decomposition theorem is in pure graph theory terms. A matroidal version is given as follows (see Seymour [35] and [37]).

Theorem 3.2.4 (Wagner [47]) Let $M$ be a graphic matroid that does not contain a minor isomorphic to $M\left(K_{5}\right)$. One of the following must hold.

(i) $M$ is a planar matroid.

(ii) $M \cong M\left(H_{8}\right)$.

(iii) $M \cong M\left(K_{3,3}\right)$.

(iv) For some $i \in\{1,2,3\}, M=M_{1} \oplus_{i} M_{2}$ is the $i$-sum of two matroids $M_{1}$ and $M_{2}$, such that both $M_{1}$ and $M_{2}$ are proper minors of $M$.

Proposition 3.2.5 (Propositions 4.2.11, 8.3.1 and 12.4.16 of [30]) Each of the following holds:

(i) The matroid $M$ is not 2-connected, if and only if for some proper non-empty subset $T$ of $E(M), M=(M \mid T) \oplus(M \mid(E \backslash T))$. Note that $M \mid T$ and $M \mid(E \backslash T)$ are both proper minors of $M$. 
(ii) The matroid $M$ is 2-connected but not 3-connected if and only if $M=M_{1} \oplus_{2} M_{2}$ for some matroids $M_{1}$ and $M_{2}$, each of which is isomorphic to a proper minor of $M$.

(iii) If $M$ is a 3-connected binary matroid and a 3-sum of $M_{1}$ and $M_{2}$, then $M_{1}$ and $M_{2}$ are isomorphic to proper minors of $M$.

\subsection{The Proofs of Theorem 3.1.8 and Corollary 3.1.9}

In view of Proposition 3.1.1, we will prove Theorem 3.1.8 by showing that $M$ has a 3 -cycle 2-cover given the assumption of the theorem. We first establish some lemmas.

Proposition 3.3.1 Each of the following holds.

(i) Each of $M\left(H_{8}\right), M^{*}\left(H_{8}\right), M\left(K_{3,3}\right), M^{*}\left(K_{3,3}\right), R_{10}, F_{7}$ has a 3-cycle 2-cover.

(ii) $F_{7}^{*}$ cannot have a 3-cycle 2-cover.

These results follow from known facts about tangential 2-block. See for example the discussion on Tutte's tangential 2-block conjecture in [7]. The results can also be verified directly in a straightforward way.

Lemma 3.3.2 Suppose that $M, M_{1}, M_{2}$ are binary matroids and that each of $M_{1}$ and $M_{2}$ has a 3-cycle 2-cover. Then each of the following holds.

(i) If $M=M_{1} \oplus M_{2}$ is a 1-sum of $M_{1}$ and $M_{2}$, then $M$ also has a 3-cycle 2-cover.

(ii) If $M=M_{1} \oplus_{2} M_{2}$ is a 2-sum of $M_{1}$ and $M_{2}$, then $M$ also has a 3-cycle 2-cover.

Proof. (i) Suppose that $M=M_{1} \oplus M_{2}$. For $k=1,2$, we assume that $M_{k}$ has a 3-cycle 2-cover, denoted as $C_{k, 1}, C_{k, 2}, C_{k, 3}$. It follows that $\left\{C_{1, i} \cup C_{2, i}: i=1,2,3\right\}$ is a 3 -cycle 2-cover of $M$. 
(ii) Now assume that $M=M_{1} \oplus_{2} M_{2}$. Denote $E\left(M_{1}\right) \cap E\left(M_{2}\right)=\{e\}$. For each $k \in\{1,2\}$, assume that $M_{k}$ has a 3 -cycle 2-cover, denoted as $C_{k 1}, C_{k 2}, C_{k 3}$. Note that by the definition of a 2-cover, $e$ appears exactly twice in each set of 3 cycles. Without loss of generality, we may assume that $e \in C_{k i}, k, i \in\{1,2\}$. Now it is easy to verify that $\left\{C_{11} \triangle C_{22}, C_{12} \triangle C_{23}, C_{13} \triangle C_{21}\right\}$ is a 3-cycle 2-cover of $M$.

Definition 3.3.3 Suppose that $M_{1}, M_{2}$ are binary matroids and that each of $Z=\left\{e_{1}, e_{2}, e_{3}\right\}=$ $E\left(M_{1}\right) \cap E\left(M_{2}\right)$ is a circuit in both $M_{1}$ and $M_{2}$. Let $L=M\left(K_{4}\right)$ with $E(L)=\left\{e_{1}, e_{2}, e_{3}, f_{1}, f_{2}, f_{3}\right\}$ such that $Z=\left\{e_{1}, e_{2}, e_{3}\right\}$ is a circuit of $L$ and $Z^{\prime}=\left\{f_{1}, f_{2}, f_{3}\right\}$ is a cocircuit of $L$, and such that $\left\{e_{j}, f_{j}\right\}$ is a perfect matching of $K_{4}$, for each $j \in\{1,2,3\}$. Define $N_{i}=M_{i} \oplus_{3} L$, for $i \in\{1,2\}$.

With the same notation in Definition 3.3.3, we observe that for each $i \in\{1,2\}$, if $Z=$ $E\left(M_{i}\right) \cap E(L)$, then $E\left(N_{i}\right) \cap E(L)=Z^{\prime}$. Moreover, for each $i \in\{1,2\}$,

$$
M_{i} \oplus_{3} L=N_{i} \text { and } N_{i} \Delta L=M_{i}
$$

By Definition 3.3.3, if $M_{1}$ and $M_{2}$ are coloopless, then $N_{1}$ and $N_{2}$ are also coloopless. The following is known (need reference).

Lemma 3.3.4 Let $N$ be a connected binary matroid with $r(N) \geq 4$, and let $Z=\left\{e_{1}, e_{2}, e_{3}\right\}$ be a 3-circuit of $N$. Then for some disjoint subsets $T_{1}, T_{2} \subseteq E(N)-Z,\left(N-T_{1}\right) / T_{2} \cong K_{4}$, where $Z$ is a 3-circuit of $\left(N-T_{1}\right) / T_{2}$.

Lemma 3.3.5 $M_{1} \oplus_{3} M_{2}=N_{1} \Delta N_{2}$.

Proof. We shall show that both sides have the same set of cycles. By Definition 2.1, for any $C \in \mathcal{C}\left(M_{1} \oplus_{3} M_{2}\right), C=C_{1} \Delta C_{2}$ with $C_{1} \in \mathcal{C}_{0}\left(M_{1}\right), C_{2} \in \mathcal{C}_{0}\left(M_{2}\right)$ and $C_{1} \cap Z=C_{2} \cap Z=$ $W$. If $W=\emptyset$, then $C \in \mathcal{C}_{0}\left(N_{1} \Delta N_{2}\right)$, by (1) in Definition 2.1. Similarly, if $W=Z$, then $C_{1} \Delta C_{2}=\left(C_{1} \Delta Z\right) \Delta\left(C_{2} \Delta Z\right) \in \mathcal{C}_{0}\left(N_{1} \Delta N_{2}\right)$. Thus we assume that $2 \geq|W| \geq 1$. 
If $|W|=1$, then without loss of generality, we assume that $W=\left\{e_{1}\right\}$. Thus $C^{\prime}=$ $\left\{e_{1}, f_{2}, f_{3}\right\}$ is a circuit of $L=M\left(K_{4}\right)$, and so by (1), $C_{1}^{\prime}=C_{1} \Delta C^{\prime} \in \mathcal{C}_{0}\left(M_{1} \oplus_{3} L\right)=\mathcal{C}_{0}\left(N_{1}\right)$, Similarly, $C_{2}^{\prime}=C_{2} \Delta C^{\prime} \in \mathcal{C}_{0}\left(N_{2}\right)$. It follows by (1) that $C_{1}^{\prime} \Delta C_{2}^{\prime} \in \mathcal{C}_{0}\left(N_{1} \Delta N_{2}\right)$. Since

$$
C_{1}^{\prime} \Delta C_{2}^{\prime}=C_{1} \Delta C^{\prime} \Delta C_{2} \Delta C^{\prime}=C_{1} \Delta C_{2},
$$

it follows that $C_{1} \Delta C_{2} \in \mathcal{C}_{0}\left(N_{1} \Delta N_{2}\right)$.

Now suppose $|W|=2$. Then without loss of generality, we assume that $W=\left\{e_{1}, e_{2}\right\}$. Thus $C^{\prime \prime}=\left\{e_{1}, e_{2}, f_{2}, f_{3}\right\}$ is a circuit of $L=M\left(K_{4}\right)$, and so by $(1), C_{1}^{\prime \prime}=C_{1} \Delta C^{\prime \prime} \in$ $\mathcal{C}_{0}\left(M_{1} \oplus_{3} L\right)=\mathcal{C}_{0}\left(N_{1}\right)$, Similarly, $C_{2}^{\prime \prime}=C_{2} \Delta C^{\prime \prime} \in \mathcal{C}_{0}\left(N_{2}\right)$. It follows by (1) that $C_{1}^{\prime \prime} \Delta C_{2}^{\prime \prime} \in$ $\mathcal{C}_{0}\left(N_{1} \Delta N_{2}\right)$. Since

$$
C_{1}^{\prime \prime} \Delta C_{2}^{\prime \prime}=C_{1} \Delta C^{\prime} \Delta C_{2} \Delta C^{\prime}=C_{1} \Delta C_{2},
$$

it follows that $C_{1} \Delta C_{2} \in \mathcal{C}_{0}\left(N_{1} \Delta N_{2}\right)$. This proves that $\mathcal{C}_{0}\left(M_{1} \oplus_{3} M_{2}\right) \subseteq \mathcal{C}_{0}\left(N_{1} \Delta N_{2}\right)$.

Conversely, pick an arbitrary $D=D_{1} \Delta D_{2} \in \mathcal{C}_{0}\left(N_{1} \Delta N_{2}\right)$, with $D_{i} \in \mathcal{C}_{0}\left(N_{i}\right), i \in\{1,2\}$. Then $D_{1} \cap Z^{\prime}=D_{2} \cap Z^{\prime}=W^{\prime}$. Since $D_{i}$ is a circuit and $Z^{\prime}$ is a cocircuit, and since $N_{i}$ is binary, $\left|W^{\prime}\right| \in\{0,2\}$. If $W^{\prime}=\emptyset$, then for each $i, D_{i} \in \mathcal{C}_{0}\left(M_{i}-Z\right)$ and so by (1), $D_{i} \in$ $\mathcal{C}_{0}\left(M_{1} \oplus_{3} M_{2}\right)$. As $D_{1} \Delta D_{2}$ is a disjoint union of $D_{1}$ and $D_{2}, D_{1} \Delta D_{2} \in \mathcal{C}_{0}\left(M_{1} \oplus_{3} M_{2}\right)$. Thus we assume that $\left|W^{\prime}\right|=2$. Without loss of generality, we may assume that $W^{\prime}=\left\{f_{1}, f_{2}\right\}$. Let $D^{\prime}=\left\{f_{1}, f_{2}, e_{3}\right\}$. Then for $i \in\{1,2\}, D^{\prime}$ is a circuit in $L$ such that $D^{\prime} \mathcal{Z}^{\prime}=\mathcal{W}^{\prime}=\mathcal{D}_{>} \mathcal{Z}^{\prime}$. It follows by (2) that $C_{i}=D^{\prime} \Delta D_{i}$ is a cycle of $M_{i}$. Moreover, as

$$
C_{1} \Delta C_{2}=\left(D^{\prime} \Delta D_{1}\right) \Delta\left(D^{\prime} \Delta D_{2}\right)=D_{1} \Delta D_{2}
$$

we conclude that $D_{1} \Delta D_{2} \in \mathcal{C}_{0}\left(M_{1} \oplus_{3} M_{2}\right)$. This proves that $\mathcal{C}_{0}\left(N_{1} \Delta N_{2}\right) \subseteq \mathcal{C}_{0}\left(M_{1} \oplus_{3} M_{2}\right)$, and so it completes the proof for this lemma.

Lemma 3.3.6 Let $M=M_{1} \oplus_{3} M_{2}$ be a 3-connected matroid. With the same notation in Definition 3.3.3, for each $i \in\{1,2\}, N_{i}$ is a minor of $M$.

Proof. By symmetry, it suffices to show that $N_{1}$ is a minor of $M$. Since $M=M_{1} \oplus_{3} M_{2}$, and since $M$ is 3-connected, $M_{2}$ is also 3-connected (need some modifications, use Seymour [35]). By Lemma 3.4, $M_{2}$ has a minor $L \cong M\left(K_{4}\right)$ such that $Z=E\left(M_{1}\right) \cap E\left(M_{2}\right)$ is a 3-circuit of $L$. It follows that $N_{1}=M_{1} \oplus_{3} L$ is a minor of $M=M_{1} \oplus_{3} M_{2}$. 
Lemma 3.3.7 Let $M=M_{1} \oplus_{3} M_{2}$ be a 3-connected matroid. With the same notation in Definition 3.3.3, if each of $N_{1}$ and $N_{2}$ has a 3-cycle 2-cover, then $M$ also has a 3-cycle 2-cover.

Proof. For any $C \in \mathcal{C}\left(N_{1}\right), C=C_{1} \Delta C_{0}$ and $C_{1} \cap Z=C_{0} \cap Z$ where $C_{1} \in \mathcal{C}\left(M_{1}\right)$ and $C_{0} \in \mathcal{C}(L)$. Therefore, $C \cap Z^{\prime}=C_{0} \cap Z^{\prime}$. Since $Z^{\prime}$ is a cocircuit of $L, C_{0} \cap Z^{\prime} \equiv 0(\bmod 2)$, and so $C \cap Z^{\prime} \equiv 0(\bmod 2)$. This implies $Z^{\prime}$ is a cocircuit of $N_{1}$. Similarly, $Z^{\prime}$ is a cocircuit of $N_{2} . Z^{\prime} \in \mathcal{C}\left(N_{1}^{*}\right) \cap \mathcal{C}\left(N_{2}^{*}\right)$.

Let $\left\{C_{j}^{i}: j=1,2,3\right\}$ be a 3-cycle 2-cover of $N_{i}$, for $i=1,2$. Since $Z^{\prime} \in \mathcal{C}\left(N_{i}^{*}\right)$, $\left|C_{i}^{j} \cap Z^{\prime}\right|=2$. Without loss of generality, we can assume that $f_{i} \notin C_{i}^{j}$, then $\left\{C_{i}^{1} \Delta C_{i}^{2}\right.$ : $i=1,2,3\}$ is a 3 -cycle 2 -cover of $M$.

proof of Theorem 3.1.8: By way of contradiction, assume Theorem 3.1.8 does not hold. Then there is a matroid $M \in E X\left(M\left(\left(P_{10}\right) \overline{3}\right), M^{*}\left(K_{5}\right)\right) \cap \mathcal{R}$ which does not admit a $4-\mathrm{NZF}$, and $|E(M)|$ is minimum.

Claim $6 M$ is 3-connected.

Otherwise, by proposition 3.2.5, for $i \in\{1,2\}, M=M_{1} \oplus_{i} M_{2}$ for some proper minors $M_{1}$ and $M_{2}$. By the minimality of $M$, both $M_{1}$ and $M_{2}$ have 3-cycle 2-covers. By Lemma 3.3.2, $M$ has a 3-cycle 2-cover, which contradicts to the choice of $M$.

By Claim 6, $M$ can't be 1 - or 2-sums. $M$ is not graphic. Otherwise since $M$ is $\left(P_{10}\right)_{\overline{3}}$-minor free, by theorem $2.1 .2, M$ admits a 4 -NZF, a contradiction. $M \nsubseteq R_{10}$ by Proposition 3.3.1. If $M$ is cographic, then $M \in E X\left(M\left(K_{5}\right)\right)$, and by Theorem 3.1.7, $M$ has a 3 -cycle 2-cover.

By Lemma 3.2.3, $M$ is graphic, cographic, $R_{10}$ or $M=M_{1} \oplus_{i} M_{2}$ for $i=1,2,3$. Therefore, $M$ has to be a 3-sum. Suppose $M=M_{1} \oplus_{3} M_{2}$. Follow definition 3.3 .3 and Lemma 3.3.6, we get $N_{i}$ is a minor of $M$ for $i=1,2$ and so $N_{i} \in E X\left(M\left(\left(P_{10}\right) \overline{3}\right), M^{*}\left(K_{5}\right)\right)$. By the minimality of $M$, both $N_{1}$ and $N_{2}$ have 3-cycle 2-covers. By Lemma 3.3.7, $M$ has a 3-cycle 2-cover. A contradiction. 
For binary matroids without $F_{7}^{*}$ minor, Seymour has established the following decomposition theorem.

Theorem 3.3.8 (Seymour [37]) Every binary matroid without $F_{7}^{*}$ minor may be obtained by means of proper 1-sums or 2-sums from regular matroids and copies of $F_{7}$.

Proof of Corollary 3.1.9: This follows from Proposition 3.3.1, Lemma 3.3.2, Theorem 3.3.8 and Theorem 3.1.8. 


\section{Chapter 4}

\section{Odd edge connectivity}

\subsection{Introduction}

It is evident that odd-edge-connectivity (see Definition 4.2.1) plays a more important role than edge-connectivity in the study of some flow and cycle cover related problems. In this paper, some earlier results in those areas are extended from $\lambda$-edge-connected graphs to odd- $\lambda$-edge-connected graphs.

For graphs with large odd-edge-connectivity, small edge-cuts (of even size) may still exist. However, there are not many results or methods developed yet to deal with small even edge-cuts. For some integer flow problems and cycle cover problems, it is pointed out in $[36,21]$ that 2-edge-cut does not exit in any smallest counterexample (to some well-known flow conjectures and cycle cover conjectures). The 3 -flow conjecture by Tutte [?] was originally proposed for odd-5-edge-connected graphs. By excluding 4-edge-cut, Kochol [23], with a sophisticated linear algebra approach, proved that 3-flow conjecture is equivalent for 5-edge-connected graphs.

In this chapter, we are to develop some general approaches to deal with small even cuts. For small even degree vertices, the vertex splitting method is to be applied and the odd-edge-connectivity is to be preserved. For non-trivial small even cuts, contrac- 
tions of internal highly connected $k$-tree blocks (as contractible configuration) are to be applied. Note that the determination and verification of contractible configurations (see Definition 4.2.8) differ significantly from each other if the corresponding graph theory properties are different. In this paper, $k$-tree blocks (see Definition 4.2.3) is to be verified as contractible configurations for some graph properties. The contractibility is either to be verified independently for each problem, or to be proved by applying some existing results (such as, a recent theorem by Barát and Thomassen [6]).

\subsubsection{Parity Subgraphs}

Definition 4.1.1 Let $H$ be a subgraph of a bridgeless graph $G=(V, E)$. $H$ is a parity subgraph of $G$ if for every vertex $v \in V(G), d_{G}(v) \equiv d_{H}(v)(\bmod 2)$.

It was proved by Tutte and Nash-Williams $[43,29]$ that every $2 k$-edge-connected graph contains at least $k$ edge-disjoint spanning trees, and proved by Itai and Rodeh [18] that every spanning tree of a graph $G$ contains a parity subgraph. The combination of these two theorems yields the following result as a direct corollary.

Theorem 4.1.2 Every $2 k$-edge-connected graph $G$ contains at least $k$ edge-disjoint parity subgraphs of $G$.

It is well-known that the search of parity subgraphs plays a central role in the proofs of some important theorems in integer flow areas. For example, the 4-flow theorem is proved by Jaeger [19] with following approach: The 4-edge-connectivity guarantees the existence of two edge-disjoint parity subgraphs (by Theorem 4.1.2) and therefore a 2-cycle cover of $G$. The 8-flow theorem was proved by Jaeger [19] with following similar approach: The 3-edge-connectivity guarantees the existence of three edge-disjoint parity subgraphs in $2 G$ and therefore, a 3-cycle cover of $G$.

Theorem 4.1.2 is to be generalized in this paper by relaxing the edge-connectivity to odd-edge-connectivity and therefore, solves an open problem proposed in [51, 12, 54]. 
We generalize the theorem and get the following

Theorem 4.1.3 Every odd- $(2 k+1)$-edge-connected graph $G$ contains at least $k$ edgedisjoint parity subgraphs of $G$.

\subsubsection{Flow Index}

Integer flow was originally introduced by Tutte [40, 41] as a generalization of map coloring problems.

Definition 4.1.4 Let $G=(V, E)$ be a graph. An ordered pair $(D, f)$ is called an integer flow of $G$ if $D$ is an orientation of $E(G)$ and $f: E(G) \rightarrow Z$, the set of integers, such that the total in-flow equals the total out-flow at every vertex. An integer flow $(D, f)$ is a $k$-flow if $|f(e)| \leq k-1$ for every edge $e$ of $G$. It is nowhere-zero if $f(e) \neq 0$ for every edge $e$ of $G$.

Circular flow, introduced in [16] as a real line extension of integer flow problem. The following is one of the many equivalent definitions for circular flows and the corresponding flow indices.

Definition 4.1.5 Let $\mathcal{D}$ be the set of all orientations of $G$, and $(A, B)$ be any edge-cut of $G$. The flow index of $G$ is defined by

$$
\phi(G)=\min _{D \in \mathcal{D}}\left\{\max _{(A, B)} \frac{\left|[A, B]_{D}\right|}{\left|[B, A]_{D}\right|}\right\}+1 .
$$

Theorem 4.1.6 (Galluccio and Goddyn, [15]) let $G$ be a 6-edge-connected graph, then the flow index $\phi(G)<4$.

We generalize the theorem above and get:

Theorem 4.1.7 Let $G$ be an odd-7-edge-connected graph, then $\phi(G)<4$. 


\subsubsection{Nowhere-zero 3-flows}

The following is an approach of Jaeger's weak 3-flow conjecture [19]. Lai and Zhang proved the following theorem

Theorem 4.1.8 ([24]) Every $4\left\lceil\log _{2} n\right\rceil$-edge-connected multigraph with $n$ vertices admits a nowhere-zero 3-flow.

As a generalization, we prove

Theorem 4.1.9 Let $G$ be a multigraph with $n$ vertices. If its odd-edge-connectivity is more than $4\left\lceil\log _{2} n\right\rceil$, then $G$ admits a nowhere-zero 3-flow.

\subsection{Notations and Lemmas}

Note: For notations not defined here, see [?] or [14].

A circuit is a connected 2-regular subgraph, while a cycle is the union of edge-disjoint circuits.

Let $G$ be a an undirected graph. Let $X \subseteq V(G)$, the set of all edges between $X$ and $Y=V(G)-X$, denoted by $(X, Y)$, is an edge-cut of $G$. If $G$ is a directed graph under an orientation, then the set of arcs from $X$ to $Y$ is denoted by $[X, Y]_{D}$.

Definition 4.2.1 A graph $G$ is said to be odd- $(2 k+1)$-edge-connected provided the size of every odd edge cut is at least $2 k+1$. The odd-edge-connectivity of $G$, denoted by $\lambda_{o}(G)$, is the size of the smallest odd edge-cut of $G$.

Tutte proposed the 3-flow conjecture [?] that every odd-5-connected graph admits a nowhere-zero 3-flow. Later Jaeger([19]) weakened the conjecture and proposed the weak 
3 -flow conjecture that there is an integer $k$ such that every $k$-edge-connected graph admits a nowhere-zero 3-flow. Also, Jaeger proved the 4-flow theorem, which is the best approach to the 3-flow conjecture so far.

Definition 4.2.2 Let $D$ be an orientation of a graph $G$, let $\Gamma$ be an Abelian group and let $f: D \rightarrow \Gamma$ ba a map. The boundary of $f$ is the map $\partial f: V(G) \rightarrow \Gamma$ where $\partial f(v)=\sum_{e \in E^{+}(v)} f(e)-\sum_{e \in E^{-}(v)} f(e)$ for each vertex $v \in V(G)$. G is said to be $\Gamma$ connected if for every $b: V(G) \rightarrow \Gamma$ with $\sum_{v \in V(G)} b(v)=0$, there exists a nowhere-zero map $f: D \rightarrow \Gamma$ with boundary $\partial f=b$.

Definition 4.2.3 Let $H$ be a subgraph of a graph $G=(V, E)$. $H$ is said to be a $k$-tree block provided $H$ is a maximal subgraph with $k$ edge-disjoint spanning trees.

The following is the key lemma of this paper and will be used in the proof of theorems 4.1.3, 4.1.7 and 4.1.9.

Lemma 4.2.4 [14, 52] Let $\left\{T_{1}, T_{2}, \cdots, T_{k}\right\}$ be a set of edge-disjoint spanning forests of a graph $G$ of maximum total size. If there is an edge $e \in E(G)-\cup_{i=1}^{k} E\left(T_{i}\right)$, then there is a k-tree block $H$ of $G$ containing e.

By counting the numbers of edges needed for $k$ edge-disjoint spanning trees, we can easily get the following corllary.

Corollary 4.2.5 If $\delta(G) \geq 2 k$, then there is a non-trivial $k$-tree block of $G$.

A polynomial algorithm was obtained in [52] for the detection of all $k$-tree-blocks of a graphs.

Lemma 4.2.6 [52] Let $G$ be a graph without $k$ edge-disjoint spanning trees, and let $H_{1}$, $H_{2}, \cdots, H_{t}$ be all $k$-tree blocks of $G$. Then

(i). $H_{i}$ and $H_{j}$ are disjoint if $i \neq j$;

(ii). $G^{\prime}=G /\left\{H_{1}, H_{2}, \cdots, H_{t}\right\}$ does not have $k$ edge-disjoint spanning trees. 
Lemma 4.2.7 [53] Let $G=(V, E)$ be a graph with odd edge connectivity $\lambda_{o}$. Assume that there is a vertex $v \in V(G)$ with degree $d$ such that $d(v) \notin\left\{2, \lambda_{o}\right\}$. Arbitrarily label the edges incident with $v$ as $\left\{e_{1}, e_{2}, \cdots, e_{d}\right\}$, then there is an integer $i \in\{1,2, \cdots, d\}$ such that the odd edge connectivity of the new graph $G^{\prime}$ obtained from $G$ by splitting $e_{i}$ and $e_{i+1}(\bmod b)$ away from $v$ remains $\lambda_{o}$.

Definition 4.2.8 A graph $H$ is a contractible configuration for property $\mathcal{P}$ if for any graph $G$ containing $H$ as a subgraph, $G$ has property $\mathcal{P}$ if and only if $G / H$ has the property.

\subsection{Proof of Theorem 4.1.3}

The following lemma is straightforward.

Lemma 4.3.1 A $k$-tree block is a contractible configuration for having $k$ edge-disjoint spanning trees.

Here, we can go further to generalize Lemma 4.3.1 for the packing problem of parity subgraphs.

Lemma 4.3.2 $A$ k-tree block is a contractible configuration for having $k$ edge-disjoint parity subgraphs.

The following definition and lemma are needed in the proof of Lemma 4.3.2.

Definition 4.3.3 Let $\vec{T}: V(G) \rightarrow Z_{2}^{k}$ be a zero-sum mapping. A $\vec{T}$-subgraph packing is a set of edge-disjoint subgraphs $\left\{P_{1}, P_{2}, \cdots, P_{k}\right\}$ such that $d_{P_{i}}(v) \equiv T_{i}(v)(\bmod 2)$ where $T_{i}(v)$ is the $i^{\text {th }}$ component of the vector $\vec{T}(v)$.

Lemma 4.3.4 If $G$ contains $k$ edge-disjoint spanning trees $T_{1}, \cdots, T_{k}$, then for any zerosum mapping $\vec{T}: V(G) \rightarrow Z_{2}^{k}, G$ has a $\vec{T}$-subgraph packing $\left\{P_{1}, \cdots, P_{k}\right\}$ such that $P_{i} \subseteq T_{i}$. 
Proof. Let $T_{1}, \cdots, T_{k}$ be $k$ edge-disjoint spanning trees. For every $i$, let

$$
S_{i}=\left\{v \in V(G) \mid \vec{T}_{i}(v) \equiv 1\right\}(\bmod 2)
$$

Note that $\left|S_{i}\right|$ is even. Partition $S_{i}$ into pairs. For each pair of vertices, there is a path in $T_{i}$. Let $P_{i}$ be the symmetric difference of these paths, then $\left\{P_{1}, \cdots, P_{k}\right\}$ is a $\vec{T}$-subgraph packing that $P_{i} \subseteq T_{i}$.

Proof of Lemma 4.3.2. Suppose a subgraph $H$ of $G$ is a $k$-tree block. If $G$ has $k$ edgedisjoint parity subgraphs $P_{1}, P_{2}, \cdots, P_{k}$, then $P_{1} / H, P_{2} / H, \cdots, P_{k} / H$ are $k$ edge-disjoint parity subgraphs of $G / H$.

Suppose $G / H$ has $k$ edge-disjoint parity subgraphs $P_{1}^{\prime}, P_{2}^{\prime}, \cdots, P_{k}^{\prime}$. For each $i \in$ $\{1,2, \cdots, k\}$, let $S_{i}=\left\{v \in V(G): d_{G \backslash E\left(P_{i}^{\prime}\right)}(v)\right.$ is odd $\}$ and $T_{i}(v)=1$ if $v \in S_{i}$ and 0 otherwise. By Lemma 4.3.4, there is a $\vec{T}$-subgraph packing $P_{1}^{\prime \prime}, \cdots, P_{k}^{\prime \prime}$ of $H$. Now $P_{1}^{\prime} \cup P_{1}^{\prime \prime}$, $P_{2}^{\prime} \cup P_{2}^{\prime \prime}, \cdots, P_{k}^{\prime} \cup P_{k}^{\prime \prime}$ are $k$ edge-disjoint parity subgraphs of $G$.

\section{Proof of Theorem 4.1.3:}

By way of contradiction, we assume $G$ is the minimum counter example with respect to the cardinality of edges, and then the cardinality of vertices.

If $G$ itself is a $k$-tree-block, then, by an observation in [18] (Itai and Rodeh), each spanning tree contains a parity subgraph. So, the minimum counterexample $G$ is not a $k$-tree-block.

We claim that

$$
\delta(G) \geq 2 k
$$

Otherwise, there is a vertex $v$ with even degree at most $2 k-1$. By Lemma 4.2.7, we can split a pair of edges away from $v$, and the resulting graph $G^{\prime}$ remains odd- $(2 k+1)$-edgeconnected. By the minimality of $G, G^{\prime}$ has $k$ edge-disjoint parity subgraphs $P_{1}, P_{2}, \cdots$, $P_{k}$. They are also parity subgraphs of $G$, which contradicts the choice of $G$.

By the inequality (4.1) and Corollary 4.2.5, there is a nontrivial $k$-tree block $H$ of $G$. Since $G / H$ has less edges and satisfies the conditions of the theorem, $G / H$ has $k$ 
edge-disjoint parity subgraphs $P_{1}^{\prime}, P_{2}^{\prime}, \cdots, P_{k}^{\prime}$. By Lemma 4.3.2, $G$ has $k$ edge-disjoint parity subgraphs. This is a contradiction and completes the proof of the theorem.

Corollary 4.3.5 Every odd-(2k+1)-connected graph $G$ has a $\left(2\left\lfloor\frac{k}{2}\right\rfloor+1\right)$ parity subgraph decomposition.

Proof. Let $P_{1}, P_{2}, \cdots, P_{k}$ be edge-disjoint parity subgraphs and $P=G \backslash \cup_{i=1}^{k} E\left(P_{i}\right)$. If $k$ is even, then $P$ is also a parity subgraph of $G$, thus we have $k+1$ edge-disjoint parity subgraphs.

\subsection{Proof of Theorem 4.1.7}

The following lemma describes the relation between integer-valued flows and orientations.

Lemma 4.4.1 [17]) Let $G$ be a bridgeless graph and $D$ be an orientation of $G$ and $a, b$ be two positive integers $(a<b)$. The following statements are equivalent.

$$
\frac{a}{b} \leq \frac{\left|[A, B]_{D}\right|}{\left|[B, A]_{D}\right|} \leq \frac{b}{a}
$$

for every edge-cut $(A, B)$ of $G$;

(2) $G$ admits a nowhere-zero integer flow $\left(D, f_{1}\right)$ such that $a \leq f_{1}(e) \leq b$ for every $e \in E(G)$.

Before the proof of Theorem 4.1.7, we are to obtain some structural characterizations about graphs $G$ with $\phi(G)=4$.

Definition 4.4.2 For an edge-cut $Q=\left\{e_{1}, e_{2}, \cdots, e_{q}\right\}=(X, Y)$ and a nowhere-zero 4flow $(D, f)$ of $G$. A balanced partition of $Q$ is a partition $Q=P_{1} \cup \cdots \cup P_{t}$ such 
that

$$
\sum_{e \in[X, Y]_{D} \cap P_{i}} f(e)=\sum_{e \in[Y, X]_{D} \cap P_{i}} f(e), \quad \forall P_{i} .
$$

If a balanced partition of $Q$ has the most number of parts among all balanced partitions, it is called a finest balanced partition. The cut $Q$ is bad with respect to $(D, f)$ if every part $P_{i}$ of a finest balanced partition is of size 4 .

Lemma 4.4.3 For a positive 4-flow $(D, f)$, an edge-cut $(X, Y)$ is bad with respect to $(D, f)$ if and only if

$$
\frac{\left|[X, Y]_{D}\right|}{\left|[Y, X]_{D}\right|}=3 \text { or } \frac{1}{3}
$$

Proof. The proof is straightforward since $(D, f)$ is a positive flow and $[X, Y]_{D} \cap P_{i} \neq \emptyset$ and $[Y, X]_{D} \cap P_{i} \neq \emptyset$ for every part $P_{i}$ of a finest balanced partition of $(X, Y)$.

Lemma 4.4.4 Let $G$ be a graph admitting a nowhere-zero 4-flow. Then $\phi(G)=4$ if and only if, for every nowhere-zero 4-flow $(D, f)$ of $G$, there is a bad cut with respect to $(D, f)$.

Proof. I. Note that every nowhere-zero 4-flow can be converted to a positive 4-flow by changing signs and reversing orientations of some edges. Hence, we are to prove the lemma for positive flows since the operation described above does not affect finest balanced partition of any edge-cut.

II. " $\Rightarrow$ ": Let $\left(D_{1}, f_{1}\right)$ be a nowhere-zero 4-flow, and $(D, f)$ be the corresponding positive 4-flow. By Lemma 4.4.1,

$$
\frac{\left|[B, A]_{D}\right|}{\left|[A, B]_{D}\right|} \leq 3
$$

for every edge-cut $(A, B)$.

$$
\text { If } \phi(G)=4 \text {, then } \phi(G) \nless 4 \text {. By Definition 4.1.5, there must be an edge cut } Q=(X, Y)
$$
with

$$
\frac{\left|[Y, X]_{D}\right|}{\left|[X, Y]_{D}\right|} \geq 3
$$


Therefore, the equality must hold for $Q=(X, Y)$. Since $(D, f)$ is a positive 4-flow of $G$, by Lemma $4.4 .3,(X, Y)$ is a bad cut with respect to $(D, f)$.

III. " $\Leftarrow$ ": Prove by contradiction. Assume that $\phi(G)<4$. Then, by Definition 4.1.5,

$$
\frac{1}{3}<\frac{\left|[X, Y]_{D_{2}}\right|}{\left|[Y, X]_{D_{2}}\right|}<3
$$

for every edge-cut $(X, Y)$ of $G$ under some orientation $D_{2}$. Furthermore, by Lemma 4.4.1, there is a positive 4 -flow $\left(D_{2}, f_{2}\right)$ that agrees with the orientation $D_{2}$. Because of $(4.2)$, $G$ has no bad-cut with respect to $\left(D_{2}, f_{2}\right)$ (by Lemma 4.4.3). This is a contradiction and completes the proof.

By Definition 4.4.2, we notice that if an edge cut $Q=(X, Y)$ is not bad with respect to a nowhere-zero 4-flow $(D, f)$, then some part $P_{i}$ of a finest balanced partition of $(X, Y)$ is of size less than 4. Therefore, either there is an edge $e \in P_{i}$ with $f(e)=2$ (if $\left|P_{i}\right|=3$ ) or there are a pair of edges $e_{1}, e_{2} \in P_{i}$ with $f\left(e_{1}\right)=f\left(e_{2}\right)$ (if $\left|P_{i}\right|=2$ ). Some of these observations are to be use very frequently in later studies.

Lemma 4.4.5 $A$ cut $Q$ is not bad with respect to a nowhere-zero 4 -flow $(D, f)$ if one of the following holds:

(1). $Q \cap E_{f= \pm 2} \neq \emptyset$;

(2). $|Q| \not \equiv 0(\bmod 4)$

The following lemma is an immediate corollary of Lemma 4.4.4 and is to used in the proof of the main theorem whenever a vertex splitting occurs.

Lemma 4.4.6 If $G^{\prime}$ is a graph obtained from $G$ by splitting a pair of incident edges, then $\phi\left(G^{\prime}\right)<4$ implies that $\phi(G)<4$.

\section{Proof of Theorem 4.1.7:}

I. By way of contradiction, let $G$ be an odd-7-edge-connected graph such that $\phi(G) \geq 4$ with least number of edges and vertices. By Corollary 4.3.5, $G$ has three edge-disjoint 
parity subgraphs. Therefore, by a theorem of Jaeger [19], it admits a nowhere-zero 4-flow $(D, f)$. That is, $\phi(G)=4$.

II. We claim that the graph $G$ itself is not a 3 -tree-block. For otherwise, let $T_{1}, T_{2}, T_{3}$ be three edge-disjoint spanning trees of $G$. Let $C_{e, T_{j}}$ be the circuit contained in $T_{j}+e$ for each edge $e \notin T_{j}$. Let $C_{1,2}=\triangle_{e \notin T_{3}} C_{e, T_{3}}$ and $C_{3}=\triangle_{e \in T_{3}} C_{e, T_{2}}$. It is easy to see that $T_{1} \cup T_{2} \subseteq C_{1,2}$ and $T_{3} \subseteq C_{3}$ and $T_{1} \cap C_{3}=\emptyset$.

For an arbitrary orientation $D$, let $\left(D, f_{1,2}\right)$ be a 2-flow of $G$ with support $C_{1,2}$ and $\left(D, f_{3}\right)$ be a 2 -flow of $G$ with support $C_{3}$. Here, $\left(D, f=2 f_{1,2}+f_{3}\right)$ is a nowhere-zero 4-flow with $T_{1} \subseteq E_{f= \pm 2}$. By Lemma 4.4.5, $\phi(G)<4$. This contradicts that $G$ is a conterexample.

III. We claim that $\delta(G) \geq 7$. Assume that there is a vertex $v$ with degree at most 6 . By Lemma 4.2.7, we can split a pair of edges away from $v$ to get a smaller odd-7-edgeconnected graph $G^{\prime}$. By the minimality of $G, \phi\left(G^{\prime}\right)<4$. By Lemma 4.4.6, $\phi(G)<4$ as well.

IV. By II, III and by Corollary 4.2.5, $G$ contains some non-trivial $k$-tree blocks. Let $\left\{H_{1}, H_{2}, \cdots, H_{a}\right\}$ be the collection of all $k$-tree blocks of $G$. By Lemma 4.2.6, $G^{\star}=$ $G /\left\{H_{1}, \cdots, H_{a}\right\}$ does not contain $k$ edge-disjoint spanning trees. By Corollary 4.2.5, $\delta\left(G^{\star}\right)=2$ or 4 .
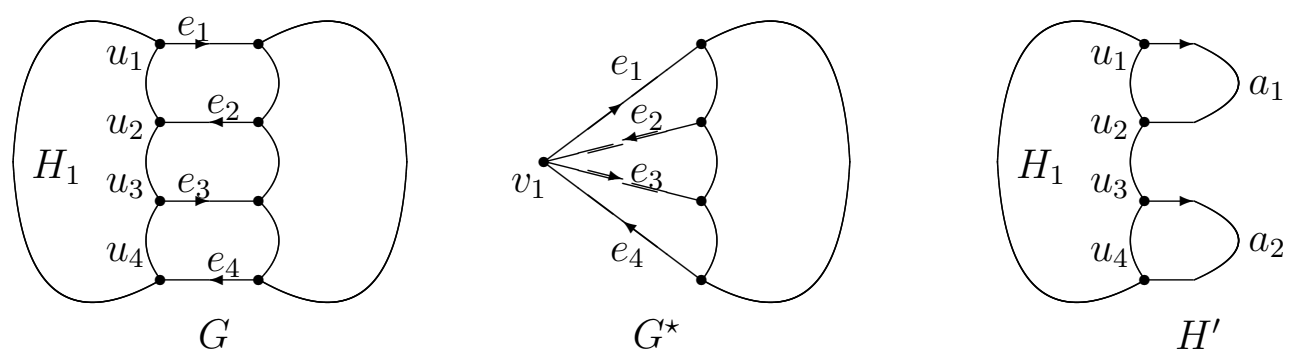

V. By IV, let $v_{1}$ be a vertex in $G^{\star}$ with degree either 2 or 4 . Let $H_{1}$ be the nontrivial $k$-tree block of $G$ corresponding to $v_{1}$ (the small degree vertex $v_{1}$ is created by contraction of $H_{1}$ since $\delta(G) \geq 6$ ). Since the smaller graph $G^{\star \star}=G / H_{1}$ remains odd-7-edge-connected, its 
flow index is less than 4 . Let $\left(D_{0}, f_{0}\right)$ be a positive 4 -flow of $G / H_{1}$ with no bad cuts. It is easy to see that the size of each part of a finest balanced partition of $E\left(v_{1}\right)$ is 2 since the edge-cut $E\left(v_{1}\right)$ is of size 2 or 4 . Without loss of generality, suppose $e_{1}, e_{3} \in E^{+}\left(v_{1}\right), e_{2}$, $e_{4} \in E^{-}\left(v_{1}\right)$ under the orientation $D_{0}$. And let $u_{i}$ be the endvertex of $e_{i}$ in $H_{1}$, for every $i=1,2,3,4$. Also, suppose $f_{0}\left(e_{1}\right)=f_{0}\left(e_{2}\right)=w_{1}, f_{0}\left(e_{3}\right)=f_{0}\left(e_{4}\right)=w_{2}$ with $w_{1} \geq w_{2} \geq 0$. (For technical reasons, if $\mid\left(V\left(H_{1}\right), V(G)-V\left(H_{1}\right) \mid=2\right.$, then $w_{2}=0$ since $e_{3}, e_{4}$ do not exist.)

Let $H^{\prime}$ be the graph obtained from $H_{1}$ by adding two arcs $a_{1}$ and $a_{2}$ that $a_{1}$ joins $u_{2}$ to $u_{1}$ and $a_{2}$ joins $u_{4}$ to $u_{3}$. Let

$$
A_{1}=\left\{a_{i} \mid w_{i} \text { is odd, } i=1,2\right\} \text { and } A_{2}=\left\{a_{i} \mid w_{i} \geq 2, i=1,2\right\} .
$$

VI. This is the final step of the proof. In this part, we are to extend the flow $\left(D_{0}, f_{0}\right)$ of $G^{\star \star}=G / H_{1}$ to the entire graph $G$ by finding a 4 -flow $\left(D^{\prime}, f^{\prime}\right)$ of $H^{\prime}$ that agrees with $a_{1}$ and $a_{2}$ in both orientations and weights. Certainly, the 4-flow $\left(D^{\prime}, f^{\prime}\right)$ of $H^{\prime}$ should not have any bad cut in $H^{\prime}$.

Since $H_{1}$ is a 3 -tree block, it has three spanning trees $T^{1}, T^{2}$ and $T^{3}$. For every edge $e \in\left(E(H)-E\left(T^{1}\right)-E\left(T^{3}\right)\right) \cup A_{1}=B$, there is a circuit $C_{e, T^{3}}$ in $T^{3} \cup e$. Let $C^{2}$ be the symmetric difference of these circuits, that is,

$$
C^{2}=\Delta_{e \in B} C_{e, T^{3}}
$$

Here $E\left(C^{2}\right) \supseteq E\left(T^{2}\right) \cup A_{1}$ and $E\left(C^{2}\right) \cap E\left(T^{1}\right)=\emptyset$.

Let $M=\left(T^{3}-C^{2}\right) \cup A_{2}$. For every edge $e \in M \cup T^{1}$, there is a fundamental circuit $C_{e, T^{2}} \subseteq T^{2} \cup e$. Let $C^{1}$ be the symmetric difference of these circuits

$$
C^{1}=\Delta_{e \in T^{1} \cup M} C_{e, T^{2}}
$$

Here $E\left(C^{1}\right) \supseteq E\left(T^{1}\right) \cup A_{2}$, and $C^{1} \cup C^{2}=E\left(H^{\prime}\right)$.

Since $C^{i}(i=1,2)$ contains a spanning tree $T^{i}$ of $H, C^{i} \backslash\left\{a_{1}, a_{2}\right\}$ is connected and, therefore, $C^{i}$ has a circuit decomposition that $a_{1}$ and $a_{2}$ are in different circuits if both $a_{1}, a_{2} \in C^{i}$. Therefore, we are able to extend the orientation of $\left\{a_{1}, a_{2}\right\}$ to all edges of 
$E\left(C^{i}\right)$ so that each member of the circuit decomposition of $C^{i}$ is oriented independently as a directed circuit (and agrees with $a_{i}$ if it contains $a_{i}$ ). Let $D^{i}$ be this eulerian orientation of the cycle $C^{i}$. We further notice that $D^{1}$ and $D^{2}$ may disagree with each other in the intersection of $C^{1}$ and $C^{2}$ (however, $D^{1}$ and $D^{2}$ agree with each other on $a_{1}$ and $a_{2}$ if any of them is contained in the intersection of $C^{1}$ and $C^{2}$ ).

Let $\left(D^{i}, f^{i}\right)$ be a non-negative 2 -flow of $H^{\prime}$ with support $C^{i}$ for each $i=1,2$.

Let $D^{3}$ be the orientation of $H^{\prime}$ obtained from $D^{1}$ and $D^{2}$ that preserves the orientation on $C^{1}$ and $C^{2} \backslash C^{1}$. Note that the orientation of $a_{1}, a_{2}$ remain the same since each $D^{i}$ agrees with each $a_{j}$ if it is in $C^{i}$. Let $\left(D^{3}, f^{2}\right)$ be the 2-flow obtained from $\left(D^{2}, f^{2}\right)$ by reversing orientations and changing signs for negative edges in the intersection of $C^{1}$ and $C^{2}$.

Then $\left(D^{3}, f^{3}=2 f^{1}+f^{2}\right)$ is a positive 4-flow and the weight of every edge in $T^{1}$ is 2 since $C^{2} \cap T^{1}=\emptyset$.

Now, the positive 4-flow $\left(D_{0}, f_{0}\right)$ of $G^{\star \star}=G / H_{1}$ can be extended to a positive 4-flow $(D, f)$ of the entire graph $G$ that agrees with both $\left(D_{0}, f_{0}\right)$ in $G^{\prime}$ and $\left(D^{3}, f^{3}\right)$ in $H_{1}$.

We only need to show $G$ does not have any bad cut with respect to $(D, f)$ (by Lemma 4.4.4). For any cut $Q$ of $G$, if $Q \cap E\left(H_{1}\right)=\emptyset$, then it is an edge cut of $G^{\star \star}$, so it is not bad. If $Q \cap E(H) \neq \emptyset$, then $Q \cap H_{1}$ is an edge-cut of $H_{1}$. Note that $T^{1}$ is a spanning tree of $H_{1}$ and $T^{1} \subseteq E_{f=2}$. Hence, there is an edge $e \in Q \cap T^{1} \subseteq E_{f=2}$. By Lemma 4.4.5, the flow index of $G$ is less than 4.That contradicts the choice of $G$.

\subsection{Proof of Theorem 4.1.9}

Lemma 4.5.1 [13, 25] Let $H$ be a $Z_{3}$-connected subgraph of a graph $G$. Then $H$ is a contractible configuration for having a nowhere-zero 3-flow.

The following two theorems was proved by Barát and Thomassen, and we will use the second one in our proof. Note these two theorems are also generalizations of Theo- 
rem 4.1.8.

Theorem 4.5.2 [6] Every $4\left\lceil\log _{2} n\right\rceil$-edge-connected multigraph with $n$ vertices is $Z_{3}$-connected.

Theorem 4.5.3 [6] Let $G$ be a multigraph with $n$ vertices. If $G$ has $2\left\lceil\log _{2} n\right\rceil$ edge-disjoint spanning trees, then $G$ is $Z_{3}$-connected.

\section{Proof of Theorem 4.1.9:}

By way of contradiction, suppose $G$ is the minimum counterexample with respect to order and size. Let $\lambda_{o}(G)=2 k+1$.

Claim: $\delta(G)>2 k$.

Otherwise, suppose $d_{G}(v) \leq 2 k$. By Lemma 4.2.7, we can split 2 edges away from $G$ to get $G^{\prime}$ where $G^{\prime}$ is still odd- $(2 k+1)$-connected. By the minimality of $G, G^{\prime}$ admits a nowhere-zero 3-flow, so does $G$.

By the Claim above and Corollary 4.2.5, there is a nontrivial $k$-tree block $H$ of $G$. Note that $G / H$ is still odd- $(2 k+1)$-connected, so it admits a nowhere-zero 3-flow.

Since $H$ has $k$ edge-disjoint spanning trees where $k>2\left\lceil\log _{2} n\right\rceil \geq 2\left\lceil\log _{2}|V(H)|\right\rceil$, by Theorem 4.5.3, $H$ is $Z_{3}$ connected. By Lemma 4.5.1, $G$ admits a nowhere-zero 3-flow. A contradiction.

\subsection{Remarks}

Note that both Theorems 4.1.7 and 4.1.9 were proved by verifying that $k$-tree-blocks are contractible configurations for these two problems. But we proved Theorem 4.1.7 without proving a similar lemma that every 3-tree-block is a contractible configuration for $\phi<4$ problem. We took the advantage of a small value $\delta\left(G^{\prime}\right)$ and paid attention only on (at most) two extra edges of a 3 -tree-block $H_{1}$ instead of all possible zero-sum boundary. This 
approach does simplify the proof of Theorem 4.1.7. However, the problem that every 3tree-block is a contractible configuration for $\phi<4$ flows remains a very interesting open problem. 


\section{Bibliography}

[1] K. Appel and W. Haken, Every planar map is four colorable, Part I: Discharging. Illinois J. Math., 21 (1977), 429-490.

[2] K. Appel, W. Haken, J. Koch, Every planar map is four colorable, Part II: Reducibility. Illinois J. Math., 21 (1977), 491-567.

[3] K. Appel and W. Haken, Every map is four colorable, Contemp. Math. AMS, 98 (1989).

[4] D. K. Arrowsmith and F. Jaeger, On the enumeration of chains in regular chain groups. J. Combin. Theory, Ser. B., 32 (1982), 75-89.

[5] J. A. Bondy and U. S. R. Murty, "Graph Theory with Applications". American Elsevier, New York, 1976.

[6] J. Barát and C. Thomassen, Claw-decompositions and Tutte-orientations, Journal of Graph Theory, 52 (2006), 135-146.

[7] T. H. Brylawski and J. G. Oxley, The Tutte Polynomial and Its Applications, in "Matroid Applications" (N. White Eds.). Cambridge University Press, Cambridge/New York, (1992).

[8] T. H. Brylawski, A decomposition for combinatorial geometries. Tran. Amer. Math. Soc. 171, (1972) 235-282.

[9] P. A. Catlin, Double cycle covers and the Petersen graph, J. Graph Theory, 13 (1989) p. $465-483$. 
[10] U. A. Celmins, On cubic graphs that do not have an edge 3-coloring, Ph.D. Thesis, University of Waterloo, Waterloo, Canada, (1984).

[11] H. H. Crapo, The Tutte polynomial. Aequaliones Math. 3 (1969), 211-229.

[12] Dean, N., Open problems in graph minors, Graph Structure Theory, Proceedings of a AMS-IMS-SIAM Joint Summer Research Conference on Graph Minors (ED. Robertson and Seymour), American Mathematical Society (1991) 677.

[13] M. DeVos, R. Xu and G. Yu, Nowhere-zero $Z_{3}$-flows through $Z_{3}$-connectivity, Disc. Math, 306 (2006) 26-30.

[14] R. Diestel, Graph Theory, third edition, Springer-Verlag, New York, 2005.

[15] A. Galluccio and L.A. Goddyn, The circular flow number of a 6-edge-connected graph is less than four, Combinatorica, 22(2002), 455-459.

[16] L. Goddyn, M. Tarsi and C.-Q. Zhang, On $(k, d)$-colorings and fractional nowhere zero flows, J. Grap Theory 28 (1998), 155-161.

[17] A. J. Hoffman, in Théorie des Graph by C. Berge, Paris (1958) p. 80.

[18] A. Itai and M. Rodeh, Covering a graph by circuits, in Automata, Languages and Programming, Lecture Notes in Computer Science 62, Springer-Verlag, Berlin, (1978) 289-299.

[19] F. Jaeger, Flows and generalized coloring theorems in graphs. J. Combinatorial Theory, Ser. B, 26 (1979), 205-216.

[20] F. Jaeger, Nowhere-zero Flow Problems, in "Selected Topics in Graph Theory" (L. Beineke and R. Wilson, Eds), Vol. 3 pp71-95. Academic Press, London/New York, 1988.

[21] F. Jaeger, A survey of the cycle double cover conjecture, in Cycles in Graphs (B. Alspach and C. Godsil, eds.), Ann. Discrete Math. 27 (1985) 1-12.

[22] Jensen and Toft, "Graph Coloring Problems", pp210-211. Wiley and Sons, New York, 1995. 
[23] M. Kochol, An equivalent version of the 3-flow conjecture. J. Combin. Theory Ser. B 83 (2001) 258-261.

[24] H.-J. Lai and C.-Q. Zhang, Nowhere-zero 3-flows of highly connected graphs, Discrete Math, 110 (1992), 179-183.

[25] H.-J. Lai, Group connectivity of 3-edge-connected chordal graphs, Graphs Combin. 16, (2000) 165-176.

[26] H.-J. Lai, Matroid Theory (in Chinese), Chinese Higher Education Press, (2002), (ISBN: 7-04-010563-2).

[27] H.-J. Lai, X. Li and H. Poon, Nowhere zero 4-flow in regular matroids, J. Graph theory, 49(2005), 196-204.

[28] K. R. Matthews, On the eulericity of a graph. J. Graph Theory, 2 (1978), 143-148.

[29] C. St. J. A. Nash-Williams, Edge-disjoint spanning trees of finite graphs, J. London Math. Soc. 36 (1961) 445-450.

[30] J. G. Oxley, "Matroid Theory". Oxford University Press, New York, 1992.

[31] N. Robertson, D. Sanders, P. Seymour and R. Thomas, The Four-Color Theorem. J. Combin. Theory, Ser. B 70 (1997), 2-44.

[32] N. Robertson, D. Sanders, P.D. Seymour and R. Thomas, Tutte's edge-colouring conjecture, J. Combin. Theory Ser. B 70 (1997), no. 1, 166-183.

[33] N. Robertson, D. Sanders, P. Seymour and R. Thomas, to appear.

[34] P. D. Seymour, Sums of circuits, in "Graph Theory and Related Topics", (Proc. Waterloo, 1977). Academic Press (1979), 341-355.

[35] P. D. Seymour, Decomposition of regular matroids. J. Combin. Theory Ser. B 28 (1980), 305-359.

[36] P. D. Seymour, Nowhere-zero 6-flows, J. Comb. Theory, B, 30 (1981) 130-135. 
[37] P. D. Seymour, Matroids and multicommodity flows. European J. Combin. Theory Ser. B. 2 (1981), 257-290.

[38] G. Szekeres, Polyhedral decompositions of cubic graphs. Bull. Austral. Math. Soc., 8 (1973), 367-387.

[39] R. Thomas and J. M. Thomson, Excluding minors in nonplanar graphs of girth at least five, Combi. Probab. Comput. 9 (2000), 573-585.

[40] W. T. Tutte, On the imbedding of linear graphs in surfaces, Proc. London Math. Soc. Ser. 2, 51 (1949) 474-483.

[41] W. T. Tutte, A contribution on the theory of chromatic polynomial, Canad. J. Math. 6 (1954) 80-91.

[42] W. T. Tutte, A homotopy theorem for matroids, I, II. Trans. Amer. Math. Soc. 88 (1958), 144-174.

[43] W. T. Tutte, On the problem of decompositing a graph into n connected factors, $J$. London Math. Soc. 36 (1961) 221-230.

[44] W. T. Tutte, Lectures on matroids. J. Res. Nat. Bur. Standards Sect. 69B. 1-47.

[45] W. T. Tutte, On the algebraic theory of graph colorings. J. Combinatorial Theory, 1 (1966), 15-50.

[46] W. T. Tutte, A geometrical version of the four color problem. Proc. Chapel Hill Conf. University of N. Carolina Press, Chapel Hill (1969) 553-560.

[47] K. Wagner, Über eine eigneschaft der ebenen komplexe. Math. Ann. 144 (1937), 570-590.

[48] P. N. Walton and D. J. A. Welsh, On the chromatic number of binary matroids. Mathematika, 27 (1980), 1-9.

[49] X. Wang, C.-Q. Zhang and T. Zhang, Nowhere-zero 4-flow in almost Petersen-minor free graphs. preprint.

[50] D. J. A. Welsh, "Matroid Theory". Academic Press, London, (1976). 
[51] West, D. Open Problems \# 06, Open Problems Columns, SIAM Activity Group Newsletter in Discrete Mathematics, Spring 1992.

[52] C.-Q. Zhang, Y. Ou, Clustering, community partition and disjoint spanning trees, manuscript.

[53] C.-Q. Zhang, Circular Flows of Nearly Eulerian Graphs and Vertex-Splitting, J. Graph Theory, 40(2002) 147-161.

[54] C.-Q. Zhang, Integer Flows and cycle covers of graphs, Marcel Dekker, New York, 1997. 